Yaramila TCHÉRÉMISSINOFF, Jean-Paul CAZES,

Christophe GILABERT, Sylvie DUCHESNE, Thibault LACHENAL, Anne LAGARRIGUE et Delphine MARET

\section{Nouvelles sépultures individuelles du Bronze ancien dans le Sud de la France : contextes et problématiques}

\begin{abstract}
Résumé
C'est la découverte de deux sépultures du Bronze ancien au sud de Toulouse (Canségala, Le Vernet, Haute-Garonne) qui est à l'origine de cet article. Depuis la fouille (2005), quelques collègues nous ont transmis d'autres découvertes qu'il a semblé utile d'intégrer et de remettre en contexte. En effet, les sépultures individuelles de cette période restent très rares en Languedoc et les sépultures en jarres étaient inconnues jusqu'ici. La première sépulture de Canségala a été réalisée pour un adulte, probablement une femme. La sépulture en jarre, qui concerne un nourrisson, était implantée à une distance d'environ $8 \mathrm{~m}$. Il avait été inséré par le siège, au sein d'un contenant dont la facture ne diffère pas de celle des productions domestiques. Quelques éléments de nature détritique (tessons, faune) issus d'une structure proche permettent de préciser le contexte de leur implantation. Leur découverte documente en effet une probable association funéraire au sein d'une aire domestique. Quant à la sépulture en jarre, elle permet l'évocation de plusieurs influences. Les seules comparaisons proches concernent des sites du Massif central (Auvergne) (Loison, 2003). Ces vases-sépultures de nourrissons peuvent être intégrés soit dans des aires domestiques soit dans des aires funéraires. Concernant les sépultures individuelles, elles demeurent rares dans le Sud de la France. En effet, la tradition chalcolithique des sépultures plurielles en coffres lithiques ou cavités y demeure vivace jusqu'au Bronze moyen. Les quelques sépultures individuelles (en silos ou en fosses spécifiques) ne semblent pas intégrées dans des cimetières, mais épouser un maillage diffus, peut-être lié à des cellules d'habitations, comme cela est attesté en Auvergne. Concernant la chronologie, les sépultures en fosses domestiques paraissent, pour l'instant, plutôt liées à la phase émergente du Bronze ancien, mais on connaît également à cette période des fosses spécifiques, clairement architecturées dans la vallée du Rhône. Les rares vases-sépultures recensés paraissent par contre tous se rapporter à un Bronze ancien plutôt évolué. Cette attribution semble être la même à plus large échelle, sur des sites où cette pratique est bien connue, comme pour le prestigieux gisement d'El Argar (phase 2, 1900 à 1600 cal. BC), (Siret et Siret, 1887; Cauwe dir., 2003), dans le Sud-Est de l'Espagne ou pour les sites sous abri de la vallée de l'Adige, dans les Alpes italiennes (Perini, 1971 et 1975; Nicolis, 1996 et 2004). Un des documents intégrés dans l'article, qui concerne un dépôt funéraire sous abri dans les Préalpes provençales (Les Baguarettes à Ménerbes, Vaucluse), permet de proposer une convergence avec les ensembles italiens, qui sont peut-être chronologiquement les plus précoces parmi les comparaisons proposées. Ceci ne suffit pas pour proposer une origine alpine du phénomène, ce domaine ne pouvant constituer qu'un relais régional par rapport
\end{abstract}


à des influences encore plus éloignées. Ce qui complique la recherche d'une éventuelle genèse est que des sépultures de jeunes enfants en jarres sont également connues localement à la fin du Néolithique... Mais une origine locale n'est pas non plus convaincante, car un important hiatus documentaire demeure pour le Campaniforme et le Bronze ancien I. À cette étape, nous pouvons juste constater que l'utilisation d'un vase comme «matrice» d'inhumation pour les jeunes enfants a pu être sporadique à la fin du Néolithique avant d'être réellement ritualisée à travers de nouvelles influences lors du développement du plein Bronze ancien.

\section{Abstract \\ The origin of this article lies in the discovery of two Early Bronze Age} graves to the south of Toulouse (Canségala, Le Vernet, Haute-Garonne). Since the excavation (2005), some colleagues have informed us of other discoveries, which it seemed interesting to integrate and set in context. Individual graves of this period remain very rare in Languedoc and burials in jars were previously unknown. The first grave at Canségala was that of an adult, probably a woman. The burial in a jar, which concerns an infant, was placed at a distance of about 8 metres. The infant had been inserted buttocks first into a container that is no different from the domestic productions. Some elements of litter (potsherds, animal bones) from a nearby structure allow the context of their implantation to be defined. Their discovery provides material concerning a possible funerary association within a domestic area. The burial in a jar allows various influences to be evoked.The only close comparisons concern some sites in the Massif Central (Auvergne) (Loison, 2003). Such infant burials in jars can be integrated in either domestic or funerary areas. As for individual graves, they remain very rare in the South of France. The Chalcolithic tradition of multiple burials in stone cists or cavities was present until the Middle Bronze Age. The few individual graves (in silos or specific pits) do not seem to be integrated in cemeteries, but seem to be vaguely organized, maybe connected to dwelling cells, as attested in Auvergne. Concerning the chronology, graves in domestic pits seem, for the moment, to be related to the emergent phase of the Early Bronze Age, but clearly "architectured" specific pits are also known for this period in the Rhone valley. The few known burials in jars all seem connected to a more advanced phase of the Early Bronze Age. This attribution seems to be the same on a wider scale on sites where this practice is well known, such as the prestigious site of El Argar (phase 2, 1900 to 1600 cal. BC), (Siret and Siret, 1887; Cauwe dir., 2003) in south-eastern Spain or for the rockshelter sites of the Adige valley, in the Italian Alps (Perini, 1971 and 1975; Nicolis, 1996 and 2004). One of the documents integrated in the article which concerns a funerary rockshelter site in the Provence Prealps (Les Baguarettes, Ménerbes, Vaucluse) allows a convergence to be proposed with the Italian series, which may chronologically be the earliest among the proposed comparisons. This is not sufficient to propose an Alpine origin for the phenomenon, as that domain could merely constitute a regional relay for influences from much farther afield. What complicates the search for a possible genesis is the fact that burials of young children in jars are also known locally at the end of the Neolithic... However a local origin is not convincing either, because there still remains an important documentary hiatus for the Bell-Beaker culture and the Early Bronze Age. At this stage, we can merely observe that the use of a jar as a "burial container" for young children could have been sporadic at the end of the Neolithic before being truly ritualized through new influences during the development of the full Early Bronze Age.

\section{PRÉSENTATION DES SÉPULTURES}

C'est la découverte de deux sépultures du Bronze ancien au sud de Toulouse (Canségala, Le Vernet,
Haute-Garonne), qui est à l'origine de cet article. Nous avions songé à réaliser une note d'actualité à leur propos, mais depuis la fouille (2005), quelques collègues nous ont informés d'autres découvertes qu'il a semblé utile d'intégrer et de mettre en contexte (fig. 1). En 

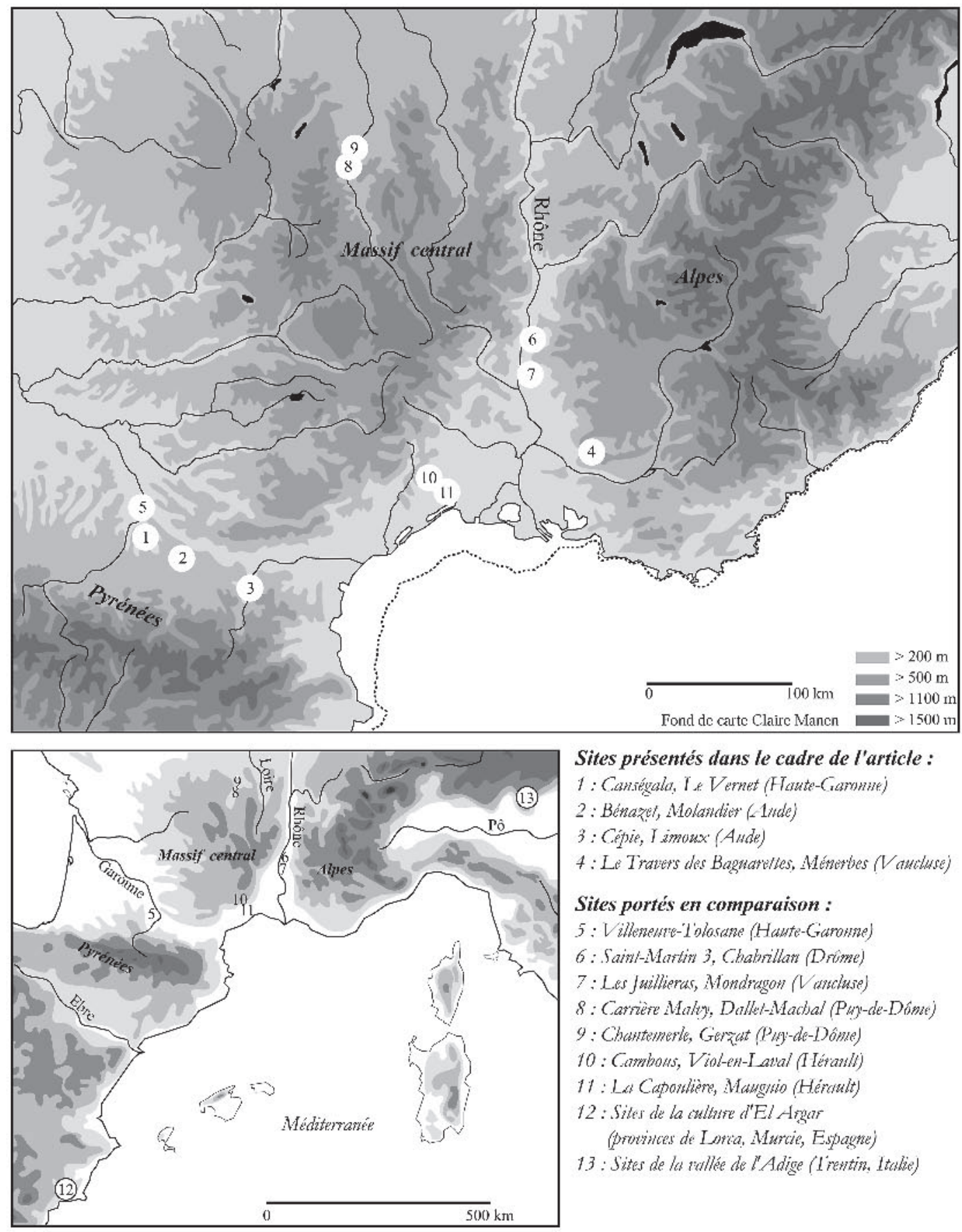

Sites présentés dans le cadre de l'article:

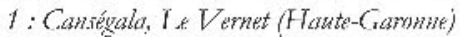

2: Bénazel, Molandior (Ande)

3: Cépie, I imoux (Alude)

4: Le Travers des Bagnarotles, Ménorbes (V auiluse)

Sites portés en comparaison :

5: Villeneune-1olosane (Huute-Guronne)

6: Sain/-Matin 3, Chabrillan (Drime)

$7:$ Les Jwillieras, Mondrugon ( $V$ artiluse)

8: Carnète Malty, Dallst-Machal (Puy-de-Dónte)

9: Chantemerle, Gerrut (Puy-de-Dônme)

10: Cambous, Violent-Laval (I Iéranll)

$11:$ La Capontière, Maugrio (Héroult)

12: Sites de la culthre d'El Argar

(provinces de Loria, Murcie, Espagne)

13: Sites de la vallee de I'Adige (Irention, Italie)

Fig. 1 - Localisation des sites (infographie : Y. Tchérémissinoff). Fig. 1 - Location of the sites.

effet, les sépultures individuelles de cette période sont très rares dans le Sud de la France, où la tradition des sépultures plus ou moins collectives en cavités ou coffres lithiques variés reste vivace. Les pratiques funéraires présentées ici se rapportent plutôt à un plein Bronze ancien, ce qui n'est pas anodin dans le contexte général. L'émergence de la sépulture en jarre a été placée au centre du propos, puisque sa manifestation n'était pas encore documentée pour cette période dans le Languedoc.

\section{Les sépultures de Canségala (Le Vernet, Haute-Garonne)}

Y. Tchérémissinoff (fouille et étude),

A. Lagarrigue (céramique), S. Duchesne (biologie) et D. Maret (observations dentaires)

La fouille au lieu-dit Canségala ou Camp Ségala (Le Vernet, Haute-Garonne) a fait suite à une évaluation réalisée sous la direction de L.-A. Lelouvier (Inrap, travaux RN 20 sud) à une quinzaine de kilomètres au 
sud de Toulouse, sur la basse terrasse de l'Ariège. Ces opérations avaient révélé différents épandages céramiques protohistoriques (Premier et Second Âges du fer), ainsi que la présence d'une tombe de nourrisson en jarre datée du Bronze ancien. Cette dernière a principalement motivé la prescription de l'intervention, ce mode sépulcral étant jusqu'alors inconnu en Languedoc, puisque les références les plus proches concernent l'Auvergne. Par ailleurs, les jarres-sépultures du Massif central étant intégrées dans de grands complexes sépulcraux ou domestiques, la découverte d'une seconde sépulture à Canségala laissait-elle envisager la présence d'un ensemble funéraire à sépultures individuelles du Bronze ancien. Malheureusement, aucune autre sépulture n'a été découverte lors du décapage extensif, ce qui est peut-être imputable à la forte érosion de la surface.

\section{La sépulture en jarre}

La jarre a été très écrêtée par la pelle mécanique, aucune variation sédimentaire ne signalant sa présence. Elle reposait de manière subhorizontale, embouchure légèrement inclinée vers le haut et orientée en direction du sud-est. Lorsque les os sont apparus, le tout a été prélevé en bloc et transféré au dépôt où nous avons procédé à la fouille.

Le défunt est un nourrisson (fig. 2 et 3).
La conservation des os est très moyenne, les régions métaphysaires sont systématiquement abîmées, mais toutes les régions anatomiques sont représentées.

Les os étaient pris dans une argile de couleur grisvert, avec inclusions de manganèse et de petites calcifications. Ils reposaient sur une séquence de ce comblement, à plus de dix centimètres de la portion de panse la plus basse dans le dépôt.

Les déplacements d'os étaient nombreux et concernaient aussi de grandes pièces comme l'humérus gauche (fig. 3 et tabl. 1, $\mathrm{n}^{\circ} 11$ ), l'ulna droite (20), la clavicule droite (24), la mandibule (18) et la scapula gauche (33). Cependant, on a aussi observé de nombreuses relations et situations logiques entre pièces, membres et secteurs anatomiques. C'est le cas d'un groupe de dents du maxillaire (2), d'un pétreux (29) et de quelques reliquats de calvaria, qui marquaient l'emplacement du crâne, logiquement situé au-dessus du gril costal contre la paroi occidentale de la jarre. Seules les côtes droites (5), en position inférieure, étaient préservées. Elles se présentaient en faisceau ouvert et vue endothoracique. Les grands os longs des membres inférieurs reposaient en fagot quelques centimètres plus à l'est, perpendiculairement aux côtes. Les extrémités distales des fémurs (15 et 28 ) et proximales des tibias (27 et 32 ) étaient toutes orientées de la même manière (au sud, vers l'embouchure de la jarre), ce qui trahit le maintien de relations partielles entre ces os et une symétrie entre

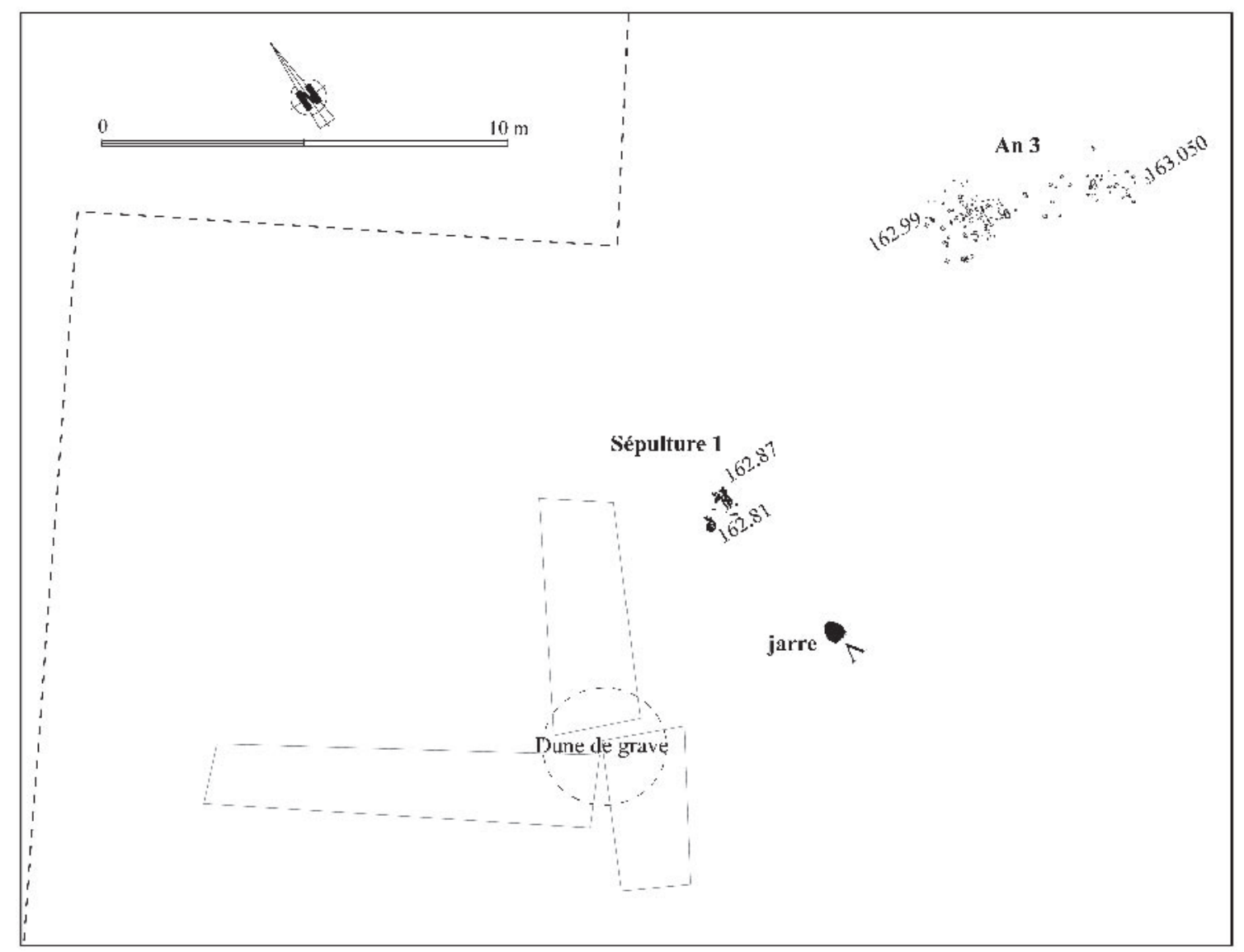

Fig. 2 - Canségala, localisation des deux sépultures (topographie : O. Onézime).

Fig. 2 - Canségala, location of the two graves. 


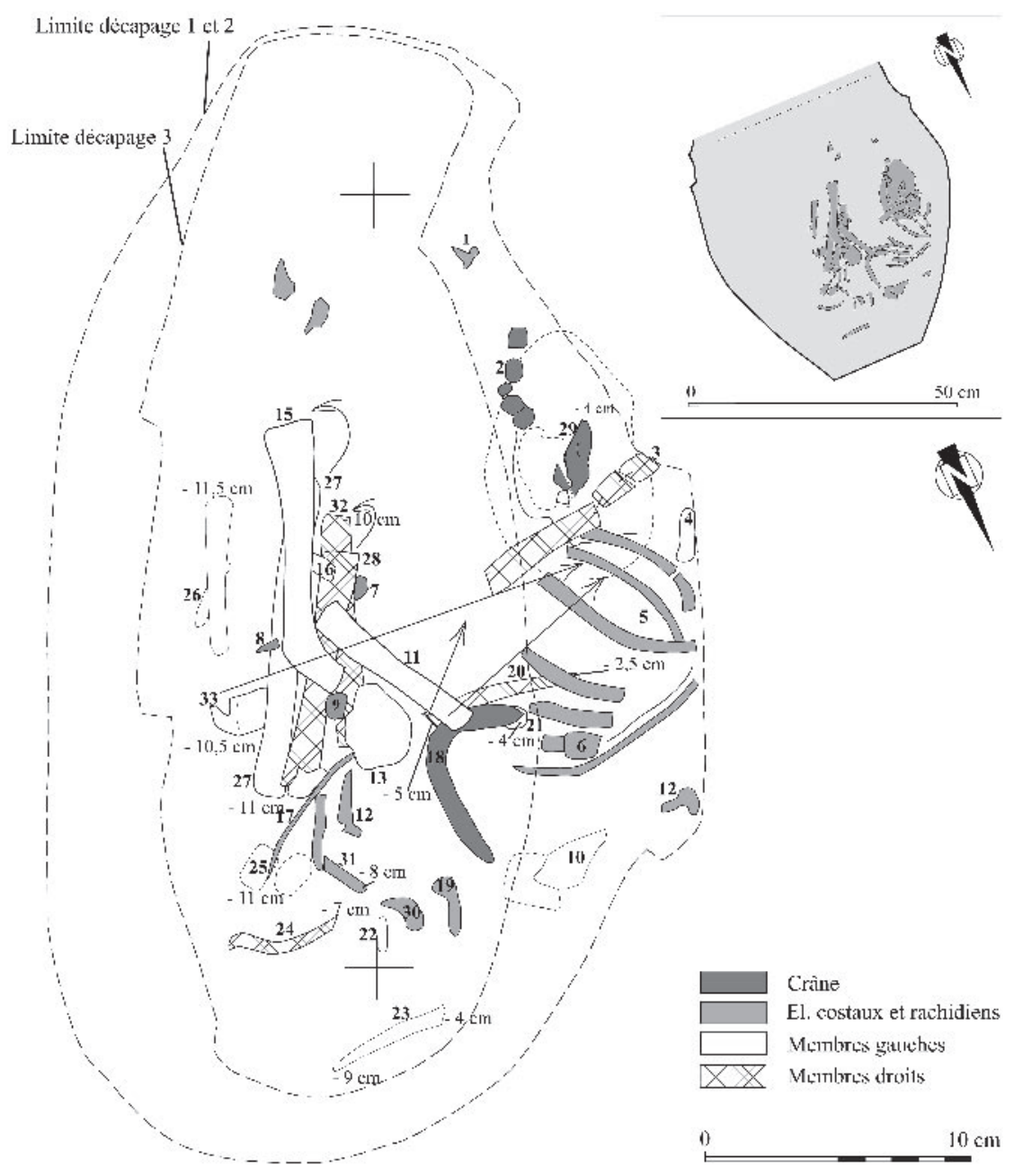

Fig. 3 - Canségala, relevé des os de la sépulture en jarre (infographie : Y. Tchérémissinoff).

Fig. 3 - Canségala, drawing of the bones of the burial in a jar.

ces membres. Le coxal gauche (13) était également convenablement localisé par rapport à ce groupe.

La position de dépôt est donc restituable. Le défunt a été introduit par le siège, en position fléchie, et déposé sur le côté droit ou sur le dos selon un axe sudouest/nord-est. L'hyperflexion observable sur les membres inférieurs est probablement imputable à un mouvement de gravité ou à une retenue des pièces en direction de l'est.

L'aspect relatif à l'état sédimentaire lors de la décomposition revêt ici une grande importance. Ces jarres sépulcrales consistent habituellement en des contenants plus ou moins hermétiques, au sein desquels les infiltrations sont généralement différées par rapport aux destructions ligamentaires. Aussi, les os se retrouvent-ils souvent amassés dans les points les plus bas des jarres, selon leurs dispositions sur les panses ou les fonds, et la lecture des positions de dépôt est alors des plus compliquées. Pour cette sépulture, le fait que des os reposent à plus de $10 \mathrm{~cm}$ du point le plus bas de la jarre et que de nombreuses relations logiques entre les os et les secteurs anatomiques permettent une lecture relativement aisée de la position constitue une sorte d'anomalie.
Par rapport à ce fait, deux propositions, au moins, peuvent être formulées. La première induit la présence initiale d'éléments périssables (emballages, support) ayant maintenu le cadavre en élévation suffisamment longtemps pour permettre aux infiltrations de le soutenir au-dessus du fond. La seconde implique un mouvement différé de la jarre en direction de l'est (vers les membres inférieurs), une bascule concomitante ou un peu postérieure à la décomposition alors que le comblement est déjà amorcé dans la portion basse du contenant. Cette dernière proposition renverrait à la présence d'un espace vide initial ou néoformé autour de la jarre. Néanmoins, dans les deux cas, il faut convenir que les infiltrations sédimentaires ont été relativement précoces.

\section{Le mobilier et l'attribution chronoculturelle}

La jarre sépulcrale est fortement déformée. Elle présente un profil globalement ovoïde dont le diamètre maximum dépasse les $460 \mathrm{~mm}$ pour une hauteur conservée de $510 \mathrm{~mm}$. Elle possède un fond plat épais, la pâte est grossière et présente de nombreuses inclusions non 


\begin{tabular}{|c|c|c|c|}
\hline $\mathbf{N}^{\circ}$ & Type & Côté & Face d'apparition \\
\hline 1 & Vertèbre cervicale & $\mathrm{G}$ & Antérieure \\
\hline 2 & Maxillaire & D & \\
\hline 3 & Humérus & $\mathrm{D}$ & Antérieure \\
\hline \multicolumn{4}{|l|}{4} \\
\hline 5 & Côtes & D & Endo-thoracique \\
\hline 6 & Vertèbre & & \\
\hline 7 & Dent & G & \\
\hline 8 & Dent & G & \\
\hline 9 & Dent & $\mathrm{D}$ & \\
\hline 10 & Ilion & $\mathrm{D}$ ? & \\
\hline 11 & Humérus & $G$ & Postérieure? \\
\hline 12 & Vertèbre thoracique & $\mathrm{D}$ & \\
\hline 13 & Coxal & G & \\
\hline \multicolumn{4}{|l|}{14} \\
\hline 15 & Fémur & G & Latéro-antérieure \\
\hline 16 & Phalange & & \\
\hline 17 & Côte & & \\
\hline 18 & Mandibule & & Inférieure \\
\hline 19 & Vertèbre thoracique & G & Inférieure \\
\hline 20 & Ulna & $\mathrm{D}$ & Postérieure \\
\hline 21 & Dent & G & \\
\hline 22 & Phalange & & \\
\hline 23 & Fibula & & \\
\hline 24 & Clavicule & $\mathrm{D}$ & Médiale \\
\hline 25 & Métatarsien & & \\
\hline 26 & Fibula & & \\
\hline 27 & Tibia & G & Latérale \\
\hline 28 & Fémur & $\mathrm{D}$ & Postéro-médiale \\
\hline 29 & Pétreux & D & Inférieure \\
\hline 30 & Vertèbre thoracique & $\mathrm{D}$ & Supéro-antérieure \\
\hline 31 & Côte & & \\
\hline 32 & Tibia & D & Postérieure \\
\hline 33 & Scapula & $\mathrm{G}$ & Antérieure \\
\hline
\end{tabular}

Tabl. 1 - Inventaire des os du sujet de la sépulture en jarre. Tabl. 1 - Inventory of the bones of the individual buried in the jar.

plastiques. Sur la panse de cet imposant récipient, quatre grands cordons arciformes, imprimés au doigt plus ou moins profondément, sont disposés deux à deux en vis-à-vis, sur deux niveaux différents. Un cordon digité horizontal a de plus été fixé près de l'embouchure du vase. Ces ajouts plastiques ont été appliqués de façon peu adhérente sur la paroi (fig. 4, $\mathrm{n}^{\circ} 3$ et fig. 5).

D'autres éléments céramiques ont été retrouvés à proximité. Il s'agit de deux éléments d'un même récipient très incomplet (fig. $4, \mathrm{n}^{\text {os }} 1$ et 2 ), qui ont peut-être été utilisés comme système de fermeture pour la jarre. D'un point de vue technique, la pâte est plutôt grossière et comprend de nombreuses inclusions non plastiques. La paroi, très érodée, est de teinte brune à orangée.

Ce vase s'ouvre par un col court vertical terminé par une lèvre arrondie (fig. $4, \mathrm{n}^{\circ} 1$ ) et porte un décor constitué d'un cordon lisse façonné en forme de fer à cheval et appliqué sur la panse (fig. $4, \mathrm{n}^{\circ} 2$ ).

La forme de la jarre sépulcrale fait partie des nombreux grands récipients à fond plat, à panse ovoïde ou parfois nettement bitronconique, qui sont fréquents durant une grande partie de l'Âge du bronze (Guilaine,
1972, p. 68, fig. 16 et 17). Le décor plastique obtenu par l'application de cordons souvent profondément imprimés est également très courant à partir du Bronze ancien. Ces grandes jarres à cordons impressionnés connaissent en effet une vaste répartition qui va de l'Italie jusqu'à l'Èbre (Guilaine, 1996, p. 63). Dans la région, on les rencontre notamment dans le Toulousain sur le site de Cassagna 3 (Pons et al., 2003, fig. 10), ou plus au sud, en Roussillon, à Montou (Claustre, 1996, fig. 2, n 11) dans deux ensembles bien datés du début de la période. En Languedoc audois, une grande jarre ornée de cordons digités disposés en guirlande est attestée sur l'habitat du Bronze ancien de Laval de la Bretonne à Monze et constitue un bon exemple de la complexité des décors qui peuvent être obtenus selon cette technique (Gascó et al., 1996, fig. 1b).

Disposés en réseaux orthogonaux et dessinant parfois de véritables résilles, ces cordons sont également présents dans une fosse ayant livré des rebuts de cuisson du Bronze moyen à Villeneuve-Tolosane (HauteGaronne). Ces caractères céramiques sont considérés comme «un legs du Bronze ancien » (Giraud et Vaquer, 1994, p. 224, fig. 3, $\mathrm{n}^{\text {os }} 9$ et 10).

Les cordons strictement arciformes ou en fer à cheval sont moins fréquents. En 1995, J. Gomez de Soto a dressé une carte de répartition des vases ornés de cordons en croissant dont les principaux exemplaires se retrouvent en basse vallée du Rhône et Languedoc oriental, Charente et Nord de la France (Gomez de Soto, 1995, p. 125, fig. 47). Dans le Sud-Ouest, un seul exemplaire est signalé à Sauveterre-la-Lémance, en Lot-et-Garonne.

En plus des exemplaires de Canségala, quelques éléments du Sud Toulousain et des Pyrénées peuvent compléter cette carte. Un cordon digité en arceau de petite taille est attesté à Villeneuve-Tolosane en HauteGaronne dans un contexte du Bronze moyen (Giraud et Vaquer, 1994, fig. 3, n 7). La grotte de la Chance à Ria dans les Pyrénées-Orientales a également livré ce type d'ornementation associé à un cordon horizontal sous la lèvre, sur un vase de forme arrondie muni de languettes de préhension. La série céramique de la grotte de la Chance est attribuée à la transition du Bronze ancien au Bronze moyen (Baills, 1995, p. 373, fig. 2, $\mathrm{n}^{\circ} 5$ ). Quelques exemples existent également dans des séries issues de cavités fouillées dans les Pyrénées centrales, comme Bédeilhac ou la grotte d'Enlène en Ariège (Rouquerol, 2004, fig. 60, $\mathrm{n}^{\circ} 4$ et fig. 83, $\mathrm{n}^{\circ} 2$ ).

Ces décors en arceaux parfois associés à un cordon fixé horizontalement sous le bord rappellent clairement la céramique d'usage du Campaniforme (jarres à cordons sous l'ouverture) comme cela a été remarqué en CentreOuest (Gomez de Soto et al., 1996, p. 500) ou dans le Nord-Ouest de la France où ce motif perdurerait jusqu'à la fin du Bronze moyen (Billard et al., 1996, p. 588).

À la vue des quelques références méridionales mentionnées concernant les cordons en arceaux, le mobilier céramique du Canségala s'inscrirait plutôt dans des productions de la seconde moitié du Bronze ancien, encore empreintes de tradition campaniforme, et vient documenter une période encore très mal connue dans cette région. 

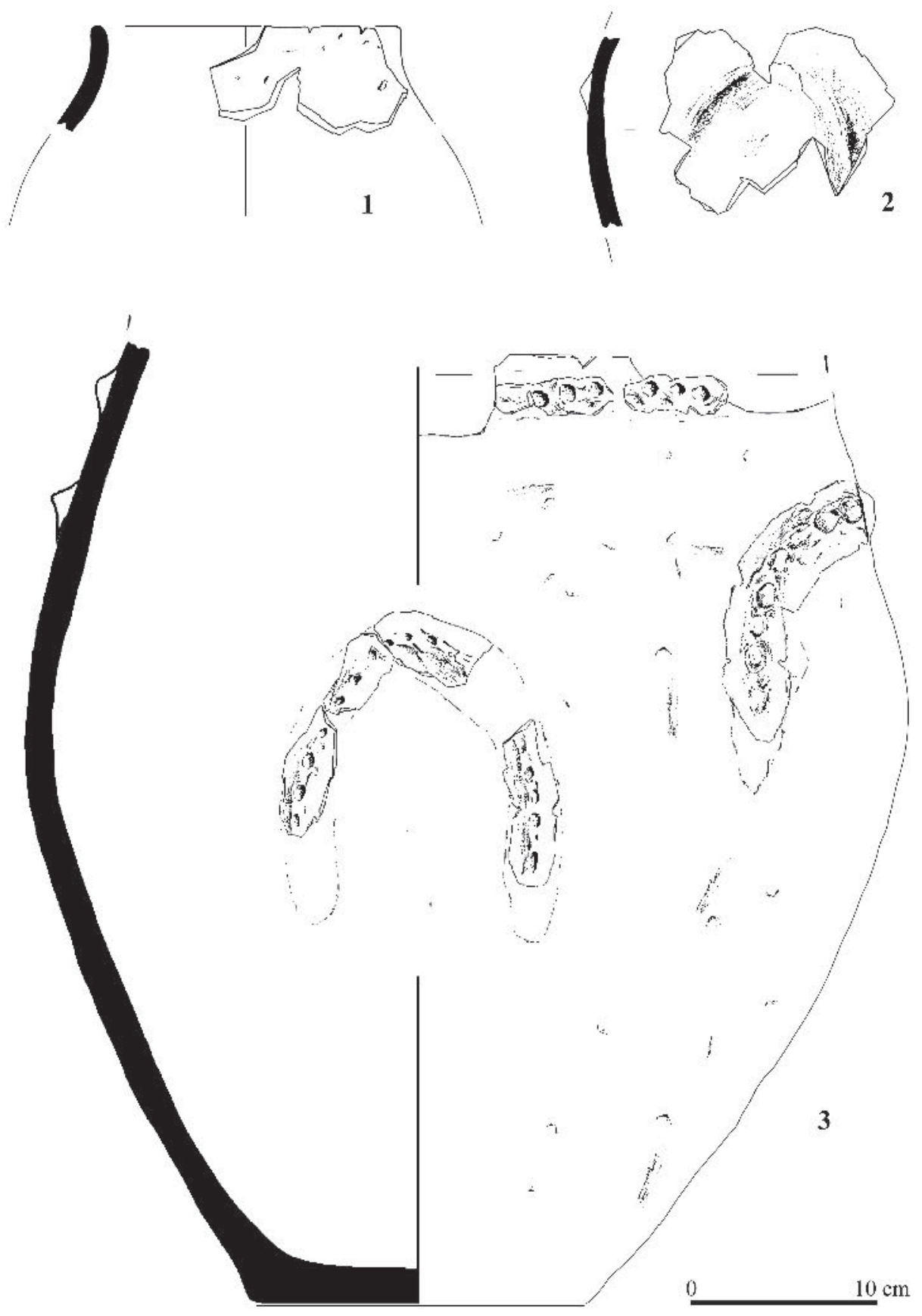

Fig. 4 - Canségala, le mobilier céramique de la sépulture en jarre (dessin : A. Lagarrigue).

Fig. 4 - Canségala, ceramic artefacts from the burial in a jar.

\section{La sépulture d'adulte}

Le crâne de cet individu avait été partiellement dégagé lors de l'évaluation.

Une relation entre cette inhumation, qui n'a pas livré de mobilier, et la tombe en jarre était suspectée en raison de leur proximité topographique. La datation ${ }^{14} \mathrm{C}, \mathrm{GrA}-35950: 3345 \pm 35$ BP : 1740 à 1520 av. n. è. ${ }^{1}$, a confirmé cette impression, bien qu'en l'absence de liens stratigraphiques lisibles, la proximité événementielle ne puisse être établie.

Le défunt est un adulte, probablement une femme d'âge avancé.
La sépulture était localisée à environ $5 \mathrm{~m}$ au nord de la jarre (fig. 2), à une altitude un peu supérieure (de $0,4 \mathrm{~m}$ ). Cette variation ne peut être interprétée en raison du profil très irrégulier de la terrasse graveleuse. Par ailleurs, aucune limite ou variation sédimentaire n'a pu être reconnue autour du squelette.

Le dégagement des os a dû être mené rapidement, car la nappe phréatique sourdait à la base du dépôt.

Le squelette est très incomplet, il manque presque tous les éléments du thorax, des avant-bras et des mains, alors que les os présentent une conservation plutôt satisfaisante. 


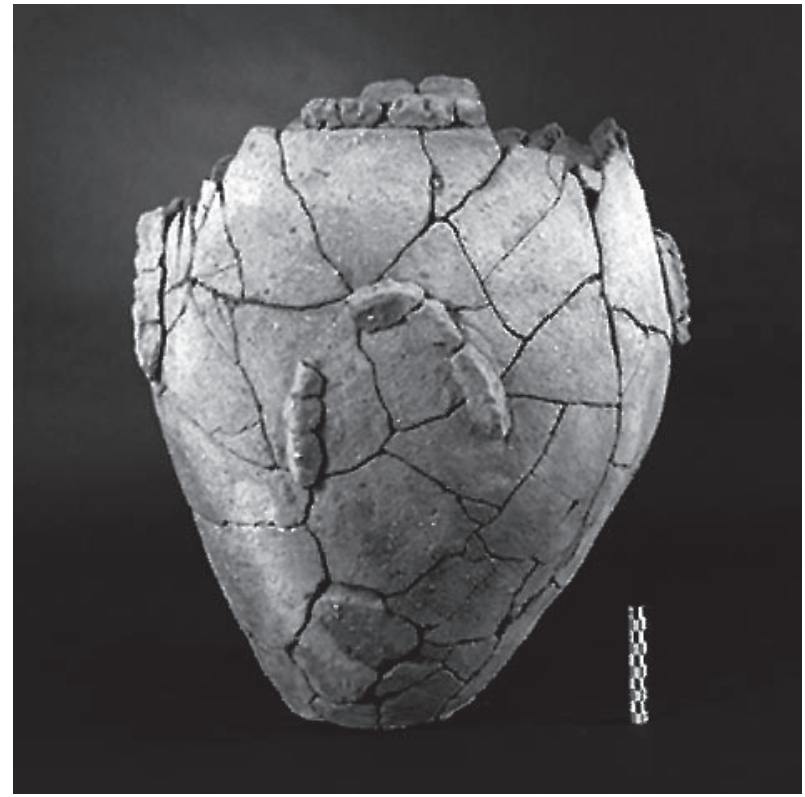

Fig. 5 - Canségala, la jarre contenant la sépulture (photo : O. Dayrens). Fig. 5-Canségala, the jar containing the burial.

Le défunt a été disposé selon un axe sud-ouest/nordest, tête au sud-ouest, les membres inférieurs étaient hyperfléchis sur le côté droit (fig. 6, 7 et 8).

Le crâne apparaissait par sa face gauche, mandibule en occlusion, il était très fragmenté. Cependant, seule la dégradation du frontal, du maxillaire et de la branche gauche de la mandibule peut être imputée à la pelle mécanique. Les deux premières vertèbres cervicales adhéraient encore à l'occipital, mais elles avaient légèrement tourné en vue antéro-latérale gauche. Une petite disjonction a d'ailleurs été remarquée entre la deuxième et troisième vertèbre, le reste de l'étage cervical demeurant en connexion et vue latérale gauche à composante postérieure. Les deux clavicules se croisaient presque à l'horizontale. La scapula gauche se présentait en vue postérieure avec une légère composante latérale, ce qui plaide peut-être en faveur d'une position initiale du tronc sur le côté droit. Cependant, cette dernière région a été trop perturbée pour que l'on puisse l'affirmer. En effet, l'absence presque totale des éléments costaux et rachidiens semble plutôt relever d'une perturbation dans cette zone, au regard de la conservation générale du squelette. D'ailleurs, l'humérus gauche, dont la moitié proximale se trouvait dans une situation normale, semble assez nettement sectionné. On a trouvé son tiers distal (il manque une petite portion de diaphyse) un peu plus bas à une trentaine de centimètres, il n'a donc pas été enlevé. C'est aussi le cas de l'autre humérus, qui s'était déplacé de plus de 0,4 m de sa position initiale, mais demeurait dans la proximité du squelette. Quelques fragments de côtes ont également été découverts à une cinquantaine de centimètres plus au nord-ouest. Il s'agit donc de déplacements importants qui concernent plusieurs grandes pièces, mais qui restent circonscrits dans la proximité de la tombe. Par contre, les os des avant-bras n'ont pas été retrouvés.

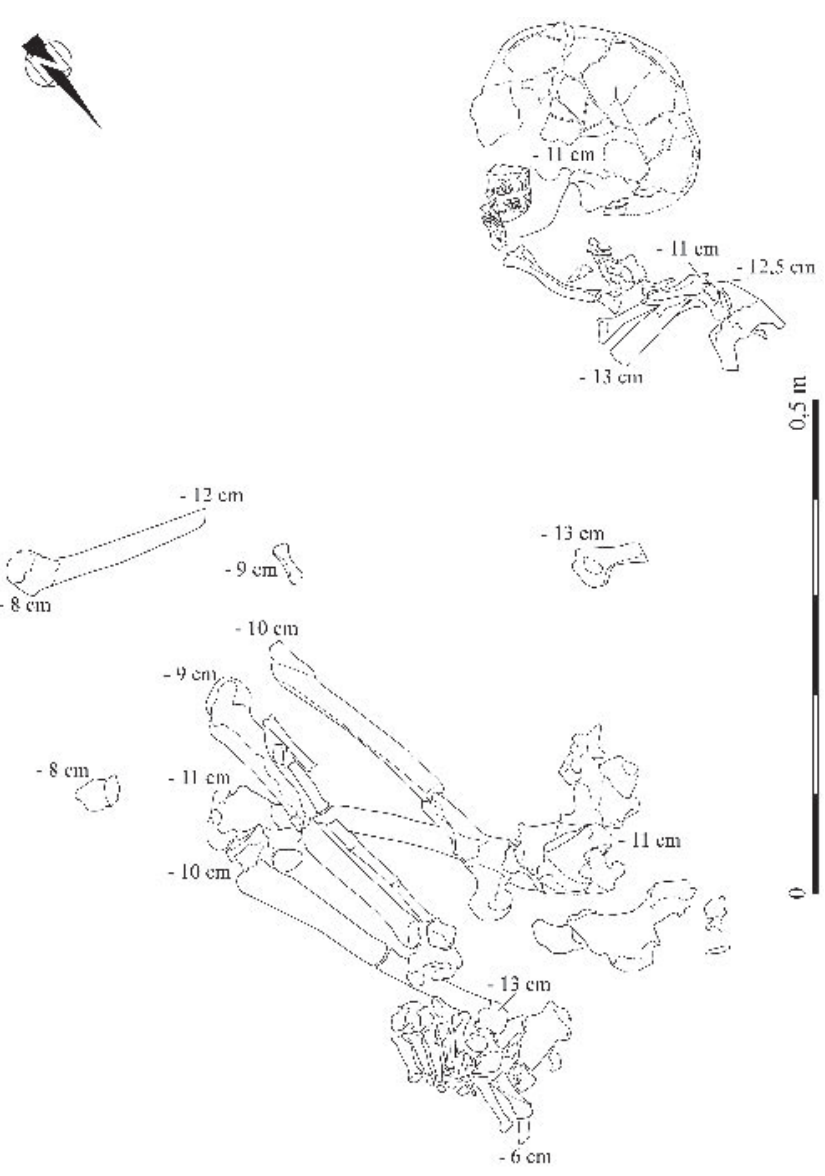

Fig. 6 - Canségala, la sépulture d'adulte (infographie : S. Puech). Fig. 6-Canségala, adult burial.

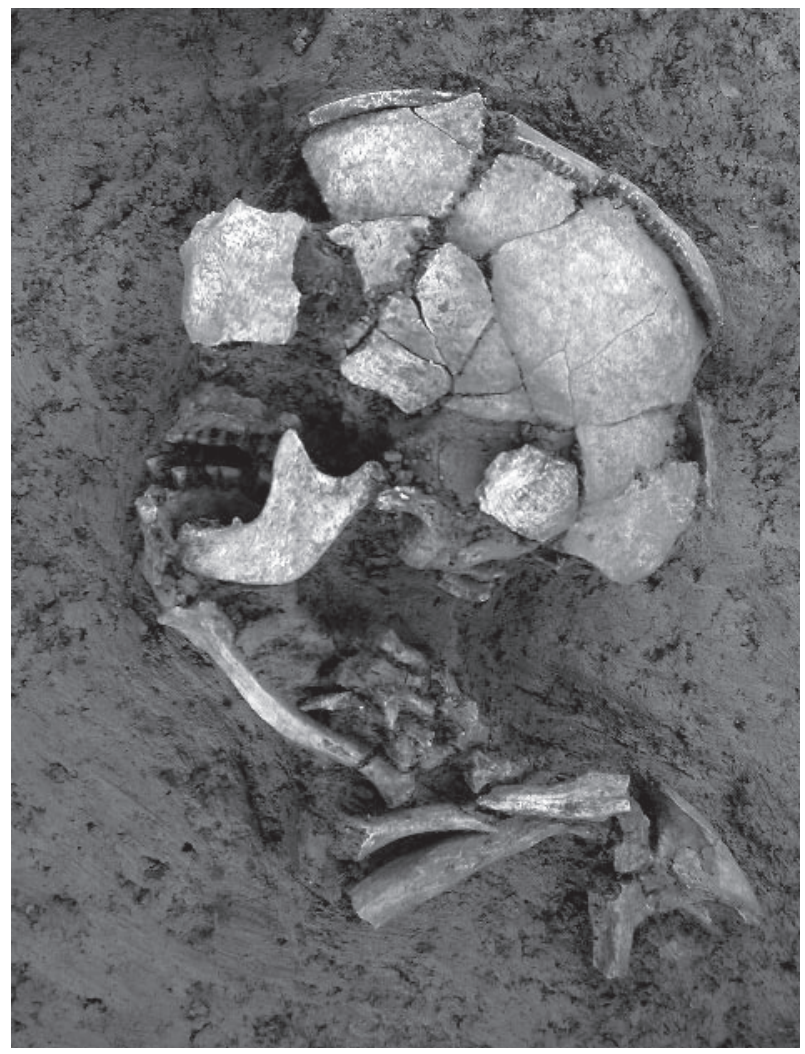

Fig. 7 - Canségala, sépulture d'adulte, détail de la partie supérieure du squelette (photo: S. Puech).

Fig. 7 - Canségala, adult burial, detail of the upper section of the skeleton. 


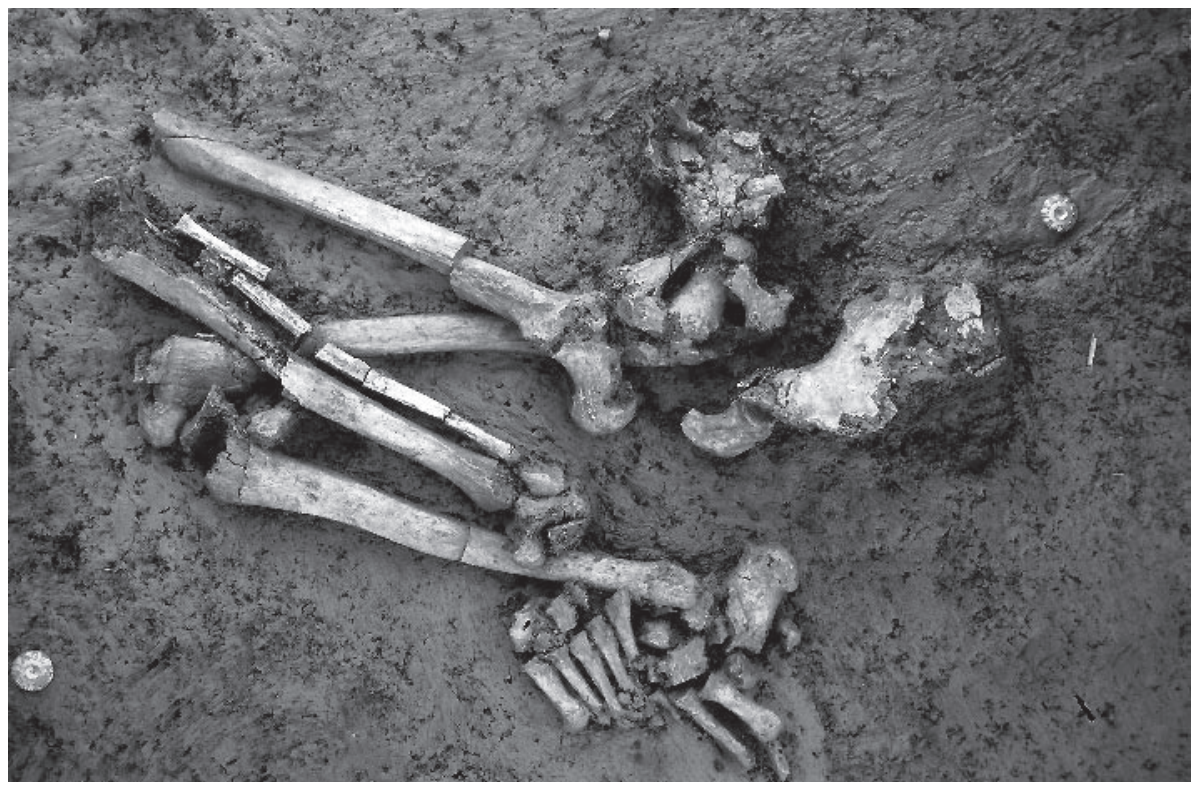

Fig. 8 - Canségala, sépulture d'adulte, détail de la partie inférieure du squelette (photo : S. Puech). Fig. 8 - Canségala, adult burial, detail of the lower section of the skeleton.

\begin{tabular}{|c|c|c|c|c|c|c|}
\hline \multirow{2}{*}{ Sépulture } & \multirow{2}{*}{$\begin{array}{c}\text { Âge dentaire } \\
\text { (Ubelaker, 1989) }\end{array}$} & \multicolumn{3}{|c|}{ Longueur diaphysaire } & \multirow{2}{*}{$\begin{array}{c}\text { Âge osseux } \\
\text { (Stloukal et Hanakova, 1978) }\end{array}$} & \multirow{2}{*}{ Âge retenu } \\
\hline & & Ulna & Fémur & Tibia & & \\
\hline Jarre & 9 mois $+/-3$ mois & est. 72 & 102 & 87 & 6 mois & 6 à 12 mois \\
\hline
\end{tabular}

Tabl. 2 - Estimation de l'âge au décès du sujet de la sépulture en jarre.

Tabl. 2 - Estimated age at the time of death of the individual buried in the jar.

Les relations du membre inférieur droit étaient assez bien préservées et les os présentaient des positions logiques, à l'exception de la patella qui avait migré à une dizaine de centimètres en direction du sud-est. Le membre opposé présentait des cassures transversales et des disjonctions plus importantes. La plus significative concerne sans aucun doute la relation de la hanche. En effet, la tête fémorale reposait à presque dix centimètres de l'acétabulum, trahissant un important mouvement d'ouverture du coxal (qui semble aussi avoir un peu tourné sur son axe en rotation interne), conjointement à la mise à plat du membre.

En conséquence, nous pensons que ce mouvement substantiel renvoie à l'absence de contrainte dans la région de la hanche gauche lors de la décomposition.

On ne perçoit cependant aucun autre élément qui pourrait évoquer un contenant rigide putrescible.

Enfin, l'origine de la perturbation (naturelle ou anthropique) lisible à l'emplacement du tronc est sans doute d'origine anecdotique.

\section{Les autres vestiges}

Il convient de signaler une concentration de mobilier subcontemporain localisée à une dizaine de mètres à l'est de la sépulture 1 (anomalie 3, fig. 2), qui est surtout composé de céramique, mais aussi d'un peu de faune domestique (étude H. Martin, Inrap), de torchis, de quelques galets et charbons. La dispersion de ces pièces s'étirait dans un axe ouest/est sur une longueur de $5 \mathrm{~m}$ et une largeur d'environ $0,5 \mathrm{~m}$. À l'extrémité ouest de cette ligne, les éléments étaient plus nombreux et formaient une tache d'un diamètre de $1,5 \mathrm{~m}$. On peut se demander si ce mobilier n'est pas issu de comblement(s) de structure(s) en creux, progressivement démantelée(s) par l'érosion.

Ce mobilier se compose de la partie supérieure d'un vase dont le bord droit orné d'impressions digitées est souligné d'un cordon imprimé également. Dans ce même lot céramique on retrouve un fragment de vase à panse vivement carénée qui ne déparerait pas en contexte du Bronze ancien ou du Bronze moyen.

La présence de ces reliquats soulève donc la question de la proximité d'une aire domestique, dont le fonctionnement pourrait être concomitant aux sépultures.

\section{Étude biologique des individus}

Le sujet immature de la jarre est un nourrisson, âgé de 6 à 12 mois (tabl. 2). La conservation de ce sujet est partielle et mauvaise : la plupart des os sont fragmentaires ou érodés. Aucune pathologie n'a été décelée.

Le sujet adulte (S.1) est assez bien conservé. Il est plutôt âgé, d'environ 40-50 ans selon les critères 


\begin{tabular}{|c|c|c|c|c|c|c|c|c|c|}
\hline Critères & \multicolumn{7}{|c|}{$\begin{array}{c}\text { Maturation osseuse } \\
\text { (Owings-Webb et Suchey, 1985) }\end{array}$} & \multicolumn{2}{|c|}{$\begin{array}{l}\text { Surface auriculaire } \\
\text { (Lovejoy et al., 1985) }\end{array}$} \\
\hline Sépulture & \multicolumn{3}{|c|}{ crête iliaque } & \multicolumn{2}{|c|}{ clavicule } & \multicolumn{2}{|c|}{ cl. d'âge } & st. coxe & cl. d'âge \\
\hline 1 & \multicolumn{3}{|c|}{ soudée } & \multicolumn{2}{|c|}{ soudée } & \multicolumn{2}{|c|}{ sup. 30 ans } & $\mathrm{V}-\mathrm{VI}$ & $40-50$ \\
\hline Critère & \multicolumn{9}{|c|}{ Usure dentaire (Lovejoy, 1985) } \\
\hline Sépulture & \multicolumn{2}{|c|}{ max. ant. } & \multicolumn{2}{|c|}{ max. post. } & \multicolumn{2}{|c|}{ mand. ant. } & \multicolumn{2}{|c|}{ mand. post. } & cl. d'âge \\
\hline 1 & $\mathrm{H}$ & $\mathrm{H}$ & $\mathrm{H}$ & $\mathrm{H}$ & $\mathrm{H}$ & $\mathrm{H}$ & I & I & $40-55$ \\
\hline
\end{tabular}

Tabl. 3 - Estimation de l'âge au décès du sujet S.1.

Tabl. 3 - Estimated age at the time of death of individual S.1.

\begin{tabular}{|c|cc|}
\hline \multirow{2}{*}{$\begin{array}{c}\text { Indices crâniens } \\
\text { (Olivier, 1960) }\end{array}$} & \multicolumn{2}{|c|}{ Sépulture 1 } \\
\cline { 2 - 3 } & Indice & Classification \\
\hline Ind. crânien horizontal & 78,3 & mésocrâne \\
\hline Ind. mixte de hauteur (droite) & 69,6 & moyen \\
\hline Ind. orbitaire & 86,5 & hypsiconque \\
\hline Capacité crânienne $\left(\mathrm{cm}^{3}\right)$ & 1359 & petite \\
\hline
\end{tabular}

Tabl. 4 - Morphologie crânienne du sujet S.1.

Tabl. 4 - Skull morphology of individual S.1.

\begin{tabular}{|c|c|c|c|c|}
\hline $\begin{array}{c}\text { Stature (en cm) } \\
\text { (Cleuvenot } \\
\text { et Houët, 1993) }\end{array}$ & mesures & moyenne & ec & intervalle \\
\hline Fémur droit & 41 & 154,6 & 3,83 & $150,8-158,5$ \\
\hline Tibia droit & 32,7 & 155,6 & 3,78 & $151,6-159,4$ \\
\hline Fibula gauche & 32,5 & 153,8 & 3,68 & $150,1-157,5$ \\
\hline
\end{tabular}

Tabl. 5 - Estimation de la stature du sujet S.1.

Tabl. 5 - Estimated height of individual S.1.

\begin{tabular}{|c|c|c|}
\hline \multirow{2}{*}{$\begin{array}{c}\text { Indices de robustesse } \\
\text { (Olivier, 1960) }\end{array}$} & \multicolumn{2}{|c|}{ Sépulture 1 } \\
\cline { 2 - 3 } & Indice & Classification (moyenne) \\
\hline Fémur & 19,5 & 20 \\
\hline Tibia & 22,9 & 20 à 21 \\
\hline
\end{tabular}

Tabl. 6 - Indices de robustesse du sujet S.1.

Tabl. 6 - Build factors for individual S.1.

\begin{tabular}{|c|c|c|c|}
\hline Sépulture 1 & \multicolumn{3}{|c|}{ Indices infra-crâniens } \\
\cline { 2 - 4 } (Olivier, 1960) & pilastrique & platymérie & cnémique \\
\hline D & 104,2 & 68,8 & 68,8 \\
\hline G & 100,0 & 69,4 & 71,9 \\
\hline Moy. & 102,0 & 69,1 & 70,3 \\
\hline Classification & faible & hyperplatymérie & eurycnémique \\
\hline
\end{tabular}

Tabl. 7 - Morphologie infra-crânienne du sujet S.1.

Tabl. 7 - Infra-cranial morphology of individual S.1.

d'âge (tabl. 3); par ailleurs, la pathologie dentaire montre de nombreuses pertes dentaires ante mortem. Les os coxaux sont très mal conservés. La détermination du sexe n'a pu être réalisée que sur deux des cinq critères morphologiques de J. Bruzek (Bruzek, 1991 et 2002), soit : la région pré-auriculaire et l'arc composé gauches, qui sont en faveur d'un sujet fémi$\operatorname{nin}$.

L'étude de la morphologie crânienne a été restreinte en raison de la mauvaise conservation de la moitié antérieure du crâne, et notamment de la face : seuls trois indices ont pu être calculés.

La boîte crânienne est moyennement arrondie (mésocrâne), la voûte est d'une hauteur moyenne et les orbites sont hautes (hypsiconques). La capacité crânienne, en retenant le sexe féminin, est petite $\left(1359 \mathrm{~cm}^{3}\right)$ (tabl. 4).

Seuls les indices des membres inférieurs ont pu être calculés, les membres supérieurs étant absents ou très fragmentaires. Le sujet, d'une taille d'environ 1,55 m (tabl. 5), est plutôt robuste. En effet, alors que les indices de robustesse féminins sont habituellement légèrement plus faibles, ils sont ici soit dans la moyenne pour le fémur, soit supérieurs pour le tibia (tabl. 6).

Le sujet présente un fémur avec un pilastre faible ou nul pour le côté gauche et une diaphyse sous les trochanters avec un aplatissement marqué d'avant en arrière (hyperplatymérie), enfin une diaphyse tibiale sans aplatissement transversal (eurycnémique) ou avec une forme moyenne pour le côté droit (tabl. 7).

Pour les caractères discrets, l'individu possède deux caractères rares : sur le crâne un os préinterpariétal et sur la mandibule un pont mylo-hyoïdien bilatéral (Hauser et De Stefano, 1989; Sjovold, 1984). Il présente également l'absence de foramen pariétal gauche, une encoche du vaste sur la patella droite, des facettes latérales d'accroupissement sur les tibias, une facette articulaire supéro-antérieure dédoublée sur le calcanéus gauche et une facette articulaire bilatérale entre le naviculaire et le cuboïde.

Les dents encore en place présentent toutes une destruction coronaire importante aboutissant même à une mise à nu pulpaire. On peut aussi noter une lésion péri-apicale sur la 33 (d'origine carieuse) associée à une forte usure coronaire. Le secteur 4 présente également une résorption osseuse.

\section{La sépulture 204 de Bénazet (Molandier, Aude)}

\section{J.-P. Cazes (dir. fouille), Y. Tchérémissinoff (étude)}

Cette sépulture a été découverte fortuitement lors de la fouille programmée d'une nécropole mérovingienne (Cazes, 2005), à la limite orientale de l'extension de cette dernière.

Le défunt reposait dans une large fosse quadrangulaire (fig. 9), apparue directement sous la semelle de 


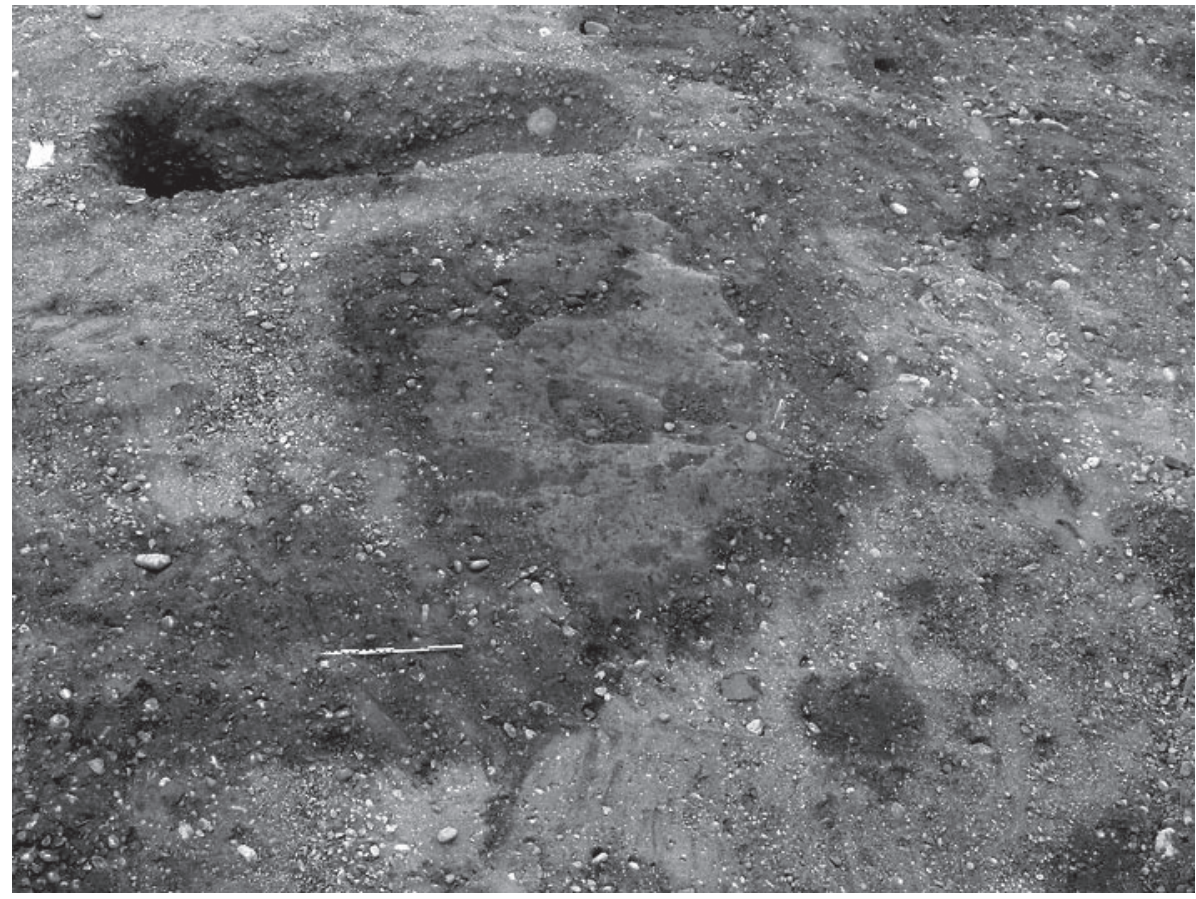

Fig. 9 - Bénazet, la fosse de la sépulture (photo : J.-P. Cazes).

Fig. 9 - Bénazet, the burial pit.

labours. Elle entamait à peine la terrasse graveleuse et n'était conservée que sur une dizaine de centimètres. Il n'a pas été possible de bien lire ses contours. On relève la présence d'une sorte de couronne disparate de galets sur un niveau. Elle pourrait constituer le reliquat d'un aménagement ou d'une signalisation un peu décentrée par rapport au dépôt. Le comblement était composé d'un limon fin, sombre, sans doute enrichi en éléments organiques. Des charbons ont d'ailleurs été retrouvés à proximité du squelette, à l'ouest, contre les galets (fig. 10). La sépulture n'a livré aucun mobilier. Une analyse ${ }^{14} \mathrm{C}$ sur os a livré la datation suivante : Poz-16802 : 3530 × 30 BP : 1950 à 1750 av. n. è., qui couvre une grande partie de la fourchette chronologique du Bronze ancien.

Le squelette est pratiquement complet, les os sont assez bien conservés.

Le défunt est un adulte de sexe masculin : l'asymétrie de l'échancrure est très nette sur le coxal droit (Bruzek, 2002). Il s'agit probablement d'un adulte âgé, car les dents présentent une forte usure occlusale, d'importants dépôts de tartre et une parodonte très marquée. L'état des surfaces sacro-pelviennes n'a pu être observé, l'un des fragments conservés présente néanmoins une forte porosité et les sutures crâniennes sont presque totalement oblitérées.

Cet individu a par ailleurs une fracture réparée sur chaque ulna. Elles ne sont pas totalement symétriques, l'ulna gauche présente un cale à mi-diaphyse, alors que la droite a été cassée à l'amorce de son tiers distal (fig. 11). Elles sont réparées dans leurs axes. Les radius n'ont pas été abîmés par le ou les impacts, on peut juste noter un aménagement de la corticale du radius gauche à proximité de la réparation. Enfin, on ne relève pas d'anomalies fonctionnelles sur les os de la main. Ces fractures pourraient être inhérentes à un geste défensif ou de protection lors d'une chute.

L'individu reposait de manière hyperfléchie, en procubitus un peu déporté sur le côté droit, dans une orientation nord-est/sud-ouest. Les membres supérieurs étaient fléchis et croisés, partiellement recouverts par le thorax, mais de manière très désaxée sur le côté gauche (bras gauche en abduction). Les membres inférieurs étaient hyperfléchis sur le côté droit, genoux relevés sous les avant-bras, pieds contre le bassin.

On constate sur la base des documents graphiques (fig. 11 à 15) que la situation réciproque des os est globalement cohérente, d'une part, et que, d'autre part, l'ensemble paraît très ramassé et soumis à pression verticale. En effet, le squelette est entièrement «mis à plat»: il n'y a pas de retenue «englobante», le tronc ou le pelvis n'ont conservé aucun volume relatif. Quelques os ont cependant migré de leur situation initiale.

C'est le cas de la mandibule qui est très déplacée vers l'avant par rapport au maxillaire, de deux métatarsiens gauches qui outrepassent le coup de pied et, peut-être, de la région de l'épaule gauche qui semble occuper une situation un peu désaxée latéralement.

La symétrie des grils costaux renvoie probablement à une absence de déplacement latéral droit important du tronc lors de sa mise à plat. Le bloc crânio-facial et les vertèbres cervicales, cependant, pourraient avoir exagérément tourné sur leur côté droit, lorsque l'on observe la rupture au niveau de la première vertèbre thoracique et la discordance entre les positions très latérales gauches des os de la tête et du cou et la 


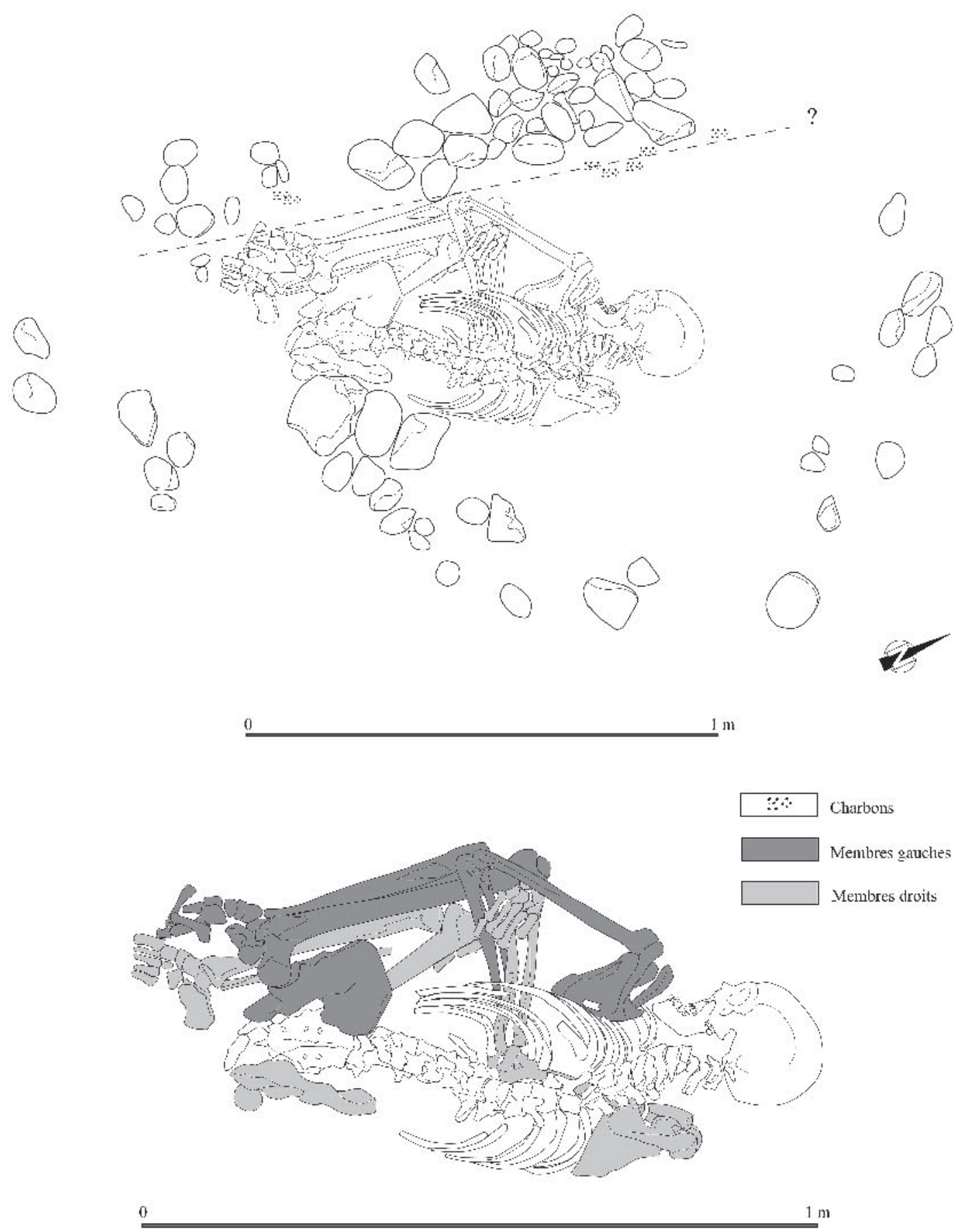

Fig. 10 - Bénazet, les lésions des ulnas (photos : Y. Tchérémissinoff).

Fig. 10 - Bénazet, injuries to the ulnae.

position très postérieure de la région du tronc. On relèvera encore le léger déboîtement de la tête fémorale gauche et la bascule latérale peut-être un peu excessive du coxal droit.

Dans le cadre de la mise à plat, la seule pesanteur du corps explique ici l'hyperflexion des membres inférieurs. En effet, aucune contrainte autre que celle liée à la gravité n'est lisible sur ce squelette.

Concernant la présence d'un contenant, les indices ostéologiques renvoyant à une protection du corps, s'ils sont ténus et très localisés (mandibule, pied gauche), existent. À notre sens, l'ampleur (la totalité) de la mise à plat constitue en soi un élément supplémentaire en faveur d'un isolement du corps de la pression sédimentaire immédiate.

À ces observations s'ajoute la présence ponctuelle de charbons, qui s'organisent de manière linéaire juste à l'ouest du squelette. Cette délinéation est également perceptible sur la bordure orientale des galets adjacents et, en l'absence d'indices symétriques, renvoie au moins à la présence initiale d'une planche à cet endroit. 


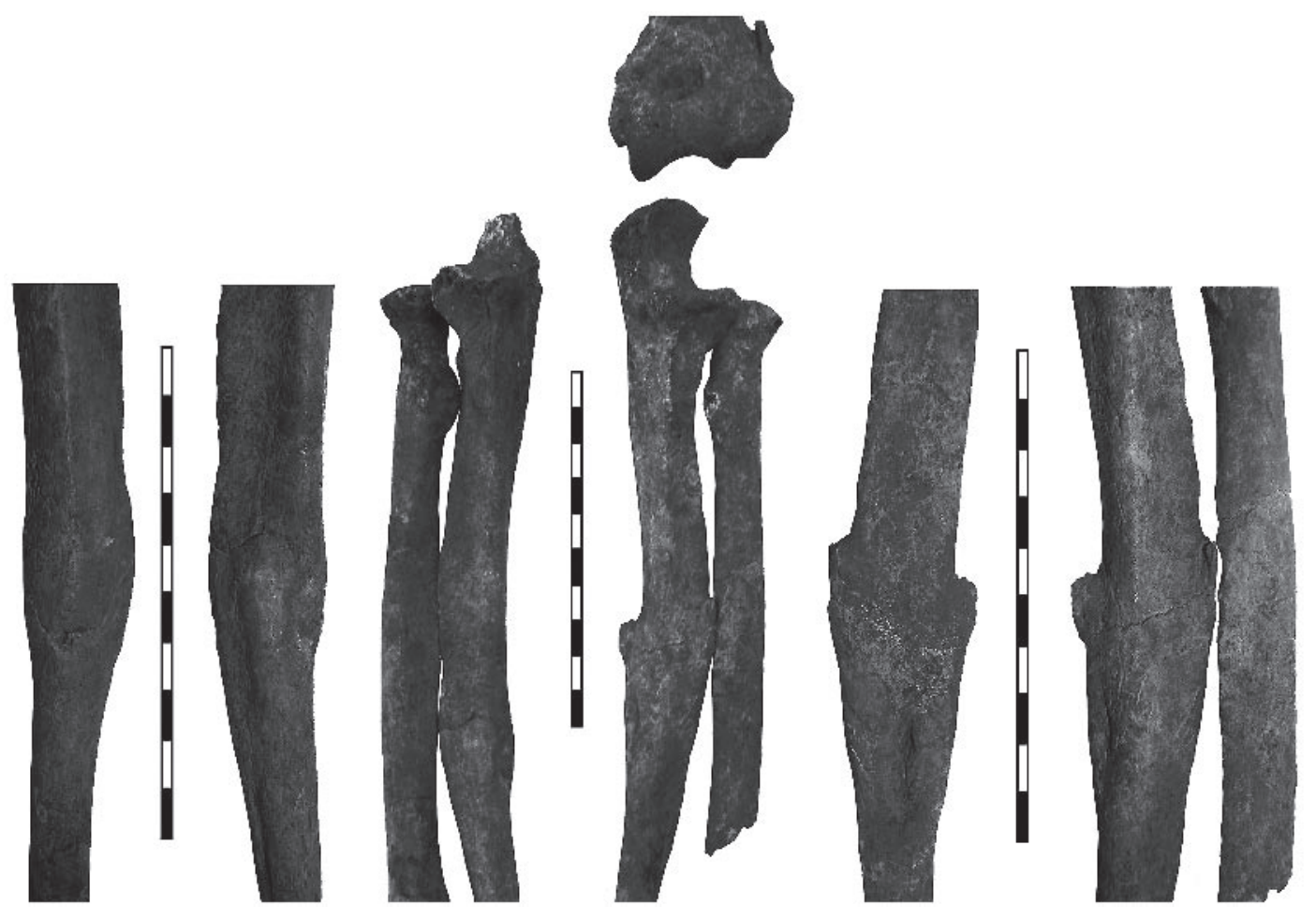

Fig. 11 - Bénazet, la sépulture (infographie : Y. Tchérémissinoff). Fig. 11 - Bénazet, the burial.

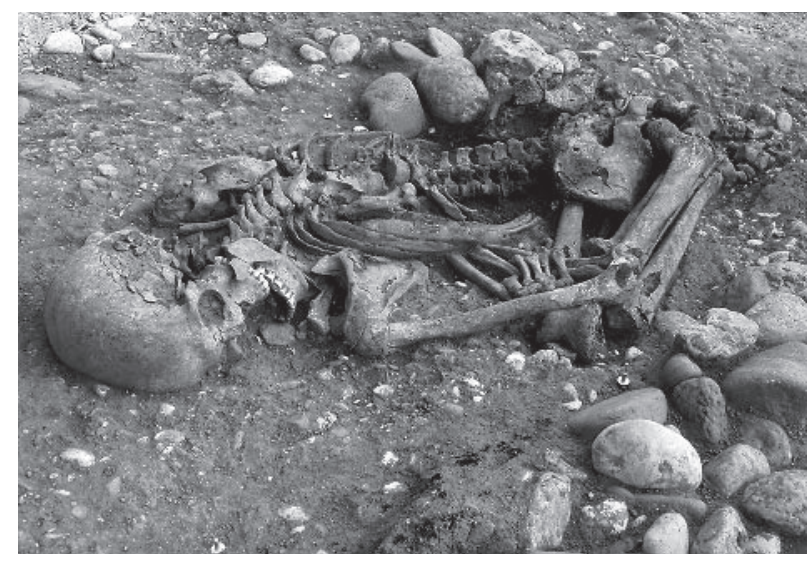

Fig. 12 - La sépulture de Bénazet vue du nord (photo : J.-P. Cazes). Fig. 12 - The Bénazet burial seen from the north.

\section{La sépulture 2014 de Cépie à Limoux (Aude) \\ P. Séjalon (dir. diagnostic) \\ et Y. Tchérémissinoff (étude)}

Cette sépulture, qui a été prélevée par nos soins lors d'une opération de diagnostic en 2001, a déjà fait l'objet d'une publication (Tchérémissinoff, 2003). En l'absence de mobilier, elle avait été rattachée par défaut au Chasséen, en raison de la proximité d'une sépulture de cette période.

La datation ${ }^{14} \mathrm{C}$, réalisée depuis, la place dans un horizon du Bronze ancien I, Ly-3589 : $3610 \pm 25$ BP : 2030 à 1890 av. n. è.

La sépulture est celle d'un adulte de sexe indéterminé. Les os, qui sont très mal conservés, affleuraient

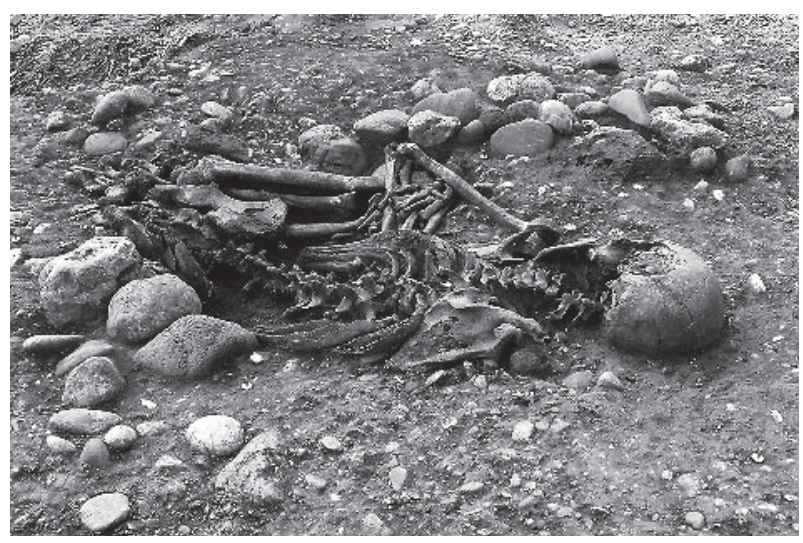

Fig. 13 - La sépulture de Bénazet vue de l'est (photo : J.-P. Cazes). Fig. 13 - The Bénazet burial seen from the east.

sous le niveau de labours. L'individu a été déposé au fond d'une fosse dont les contours n'ont pu être appréciés car elle n'entame pas la terrasse graveleuse.

Le sujet reposait sur le côté gauche, membres supérieurs et inférieurs hyperfléchis (fig. 16).

Les os de la main gauche masquaient partiellement ceux de la droite. Ils présentaient des relations strictes (à l'exception du premier et du deuxième rayon), leur position évoquant celle du maintien d'un violon. La main droite offrait une position presque analogue, produisant une sorte d'effet miroir, mais les doigts étaient davantage fléchis.

La position de dépôt est très lisible puisque aucun os n'était sorti de l'espace corporel et que toutes les 


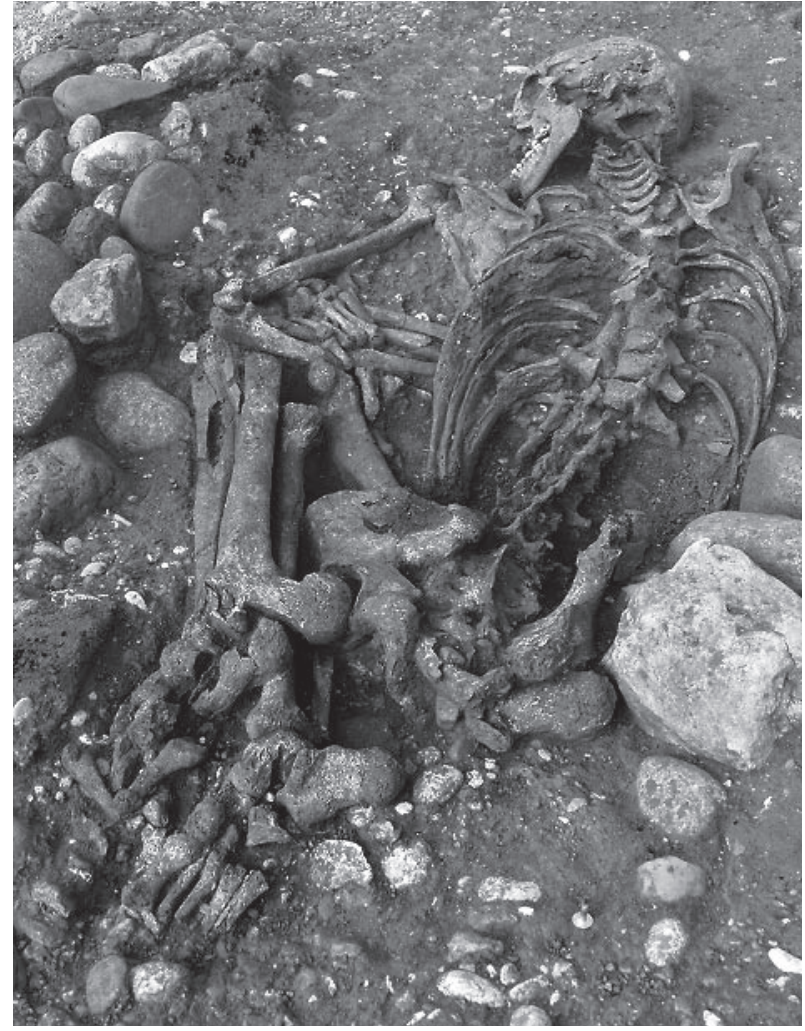

Fig. 14 - La sépulture de Bénazet vue du sud (photo : J.-P. Cazes). Fig. 14 - The Bénazet burial seen from the south.

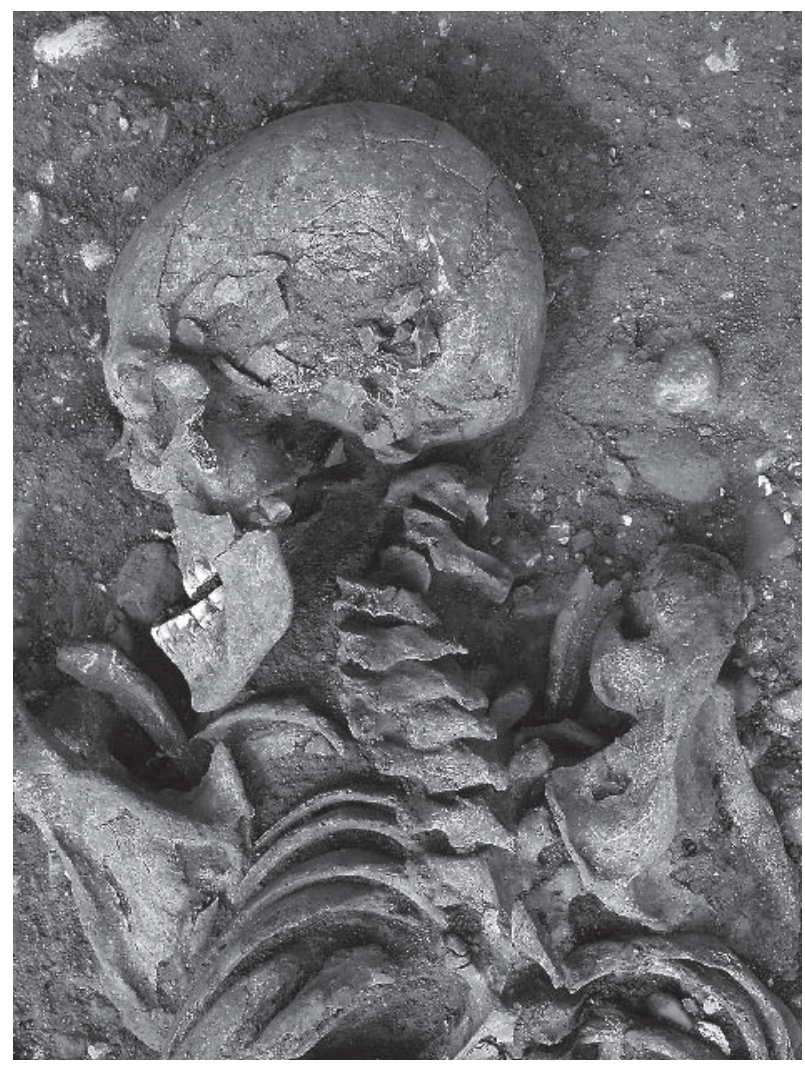

Fig. 15 - La sépulture de Bénazet, détail (photo : J.-P. Cazes). Fig. 15 - The Bénazet burial, detail.

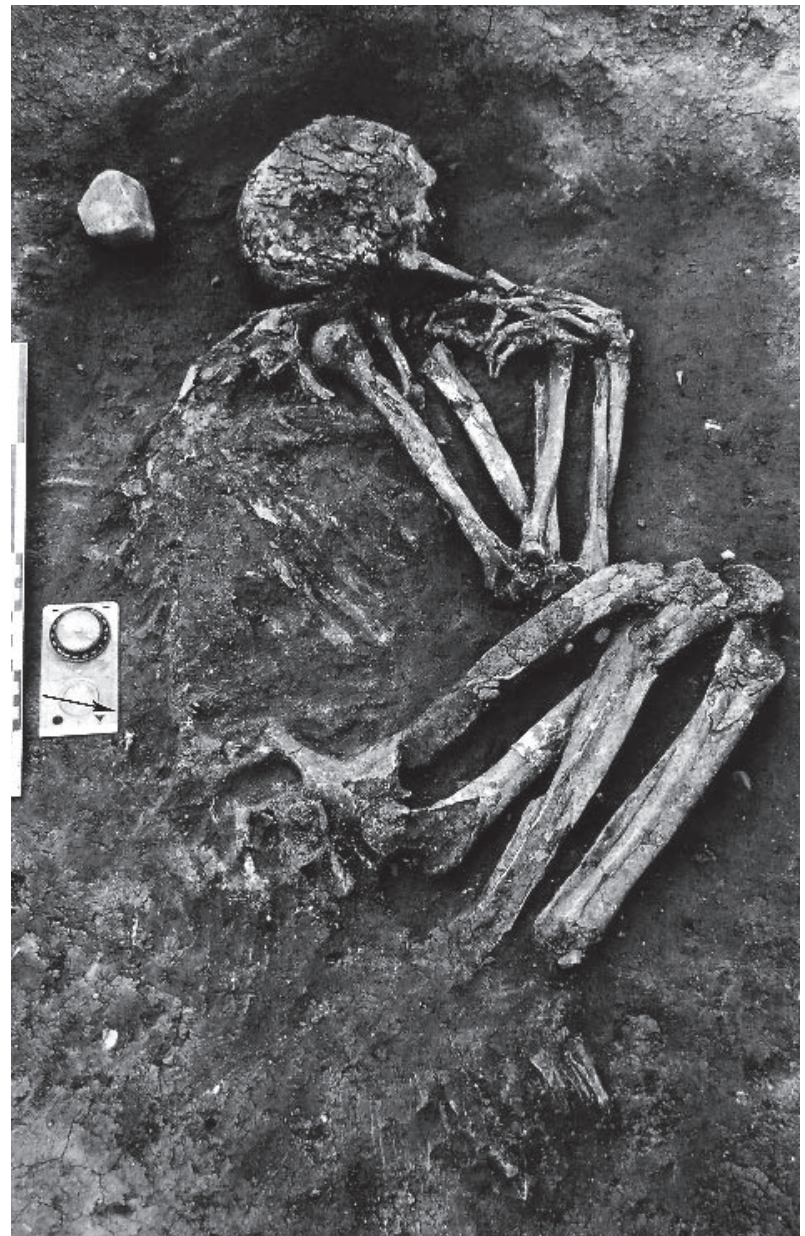

Fig. 16 - Cépie, la sépulture 2014 (photo : Y. Tchérémissinoff). Fig. 16 - Cépie, burial 2014.

pièces, même les plus petites, occupaient des positions conformes les unes par rapport aux autres. Cette observation va plutôt dans le sens d'une décomposition en espace colmaté mais il n'est pas possible d'aller audelà.

\section{La «sépulture» en vase du Travers des Baguarettes (Ménerbes, Vaucluse) \\ C. Gilabert (dir. fouille), T. Lachenal (céramique), A. Thomann (anthropologie)}

Les données concernant ce dépôt sont un peu ambiguës (Gilabert et al., 2006). Néanmoins, elles s'inscrivent probablement dans des pratiques documentées dans les Alpes italiennes, sur lesquelles nous reviendrons à l'occasion des comparaisons.

Cette «sépulture » a été trouvée au sein d'une petite cavité intégrée dans l'espace collinéen des contreforts alpins du nord du Lubéron, qui s'ouvre sur une légère pente reprise en terrasse par des travaux récents.

La conservation du site était très mauvaise. Aucune stratigraphie n'était conservée, car les sédiments marneux piégés se sont régulièrement érodés par gravité. Seul un replat localisé contre la paroi rocheuse en 
retrait de la pente a été épargné par les différents facteurs naturels et humains à l'origine de la dégradation du site.

C'est sur ce replat d'environ $4 \mathrm{~m}^{2}$, à l'abri relatif d'un léger surplomb de la paroi, qu'a été découvert un grand vase brisé en deux parties. Aucune limite éventuelle d'enfouissement n'a pu être remarquée, le vase semblait juste déposé à même le sol. Sur le plan (fig. 17), quelques pierres paraissent l'entourer, notamment une grande dalle, mais elles sont probablement issues de décollements de paroi postérieurs au dépôt.

Les deux parties du vase n'étaient pas en position primaire. Elles sont en effet situées à plus de $20 \mathrm{~cm}$ l'une de l'autre, chacune des parties présentant une orientation divergente dans l'axe nord-ouest/sud-est (fig. 18). L'origine de la fracture et du déplacement des deux parties de cette pièce ne peut être établie car le gisement a été trop perturbé. Cependant, cette cassure étant très franche, le vase n'ayant pas éclaté en de multiples tessons dispersés, il était conséquemment déjà peut-être comblé.

Différents vestiges ont été retrouvés dans le comblement résiduel (sablo-limoneux) des portions de vase ou dans leur proximité directe, en l'occurrence à moins de $20 \mathrm{~cm}$. Le reste de la surface conservée n'ayant rien livré, il s'agit donc de vestiges très localisés.

Ce mobilier se compose :

- d'un petit éclat allongé, entier de $2 \mathrm{~cm}$ de longueur pour $0,9 \mathrm{~cm}$ de largeur en silex blond bédoulien présentant une légère patine blanchâtre (inf. S. Renault);

- de quelques os de faune (quatre pièces, lagomorphe, batracien ou reptile et une dent fossile de requin (inf. V. Forest);

- de divers ossements humains.

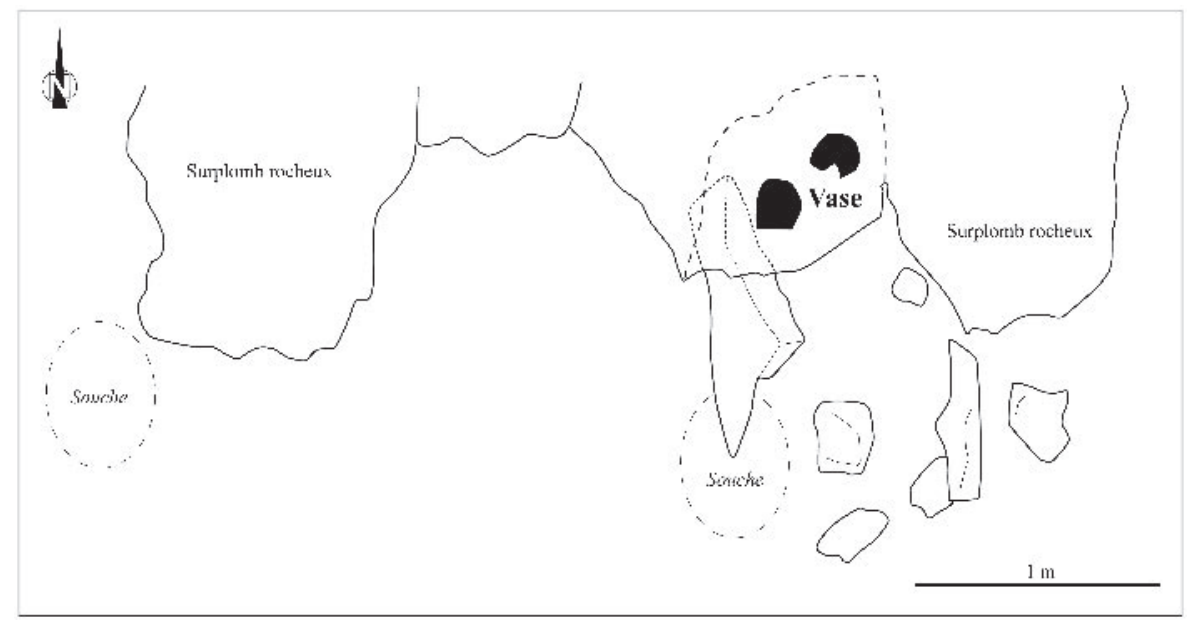

Fig. 17 - Les Baguarettes, plan de l'abri (relevé, dessin : C. Gilabert).

Fig. 17 - Les Baguarettes, plan of the shelter.

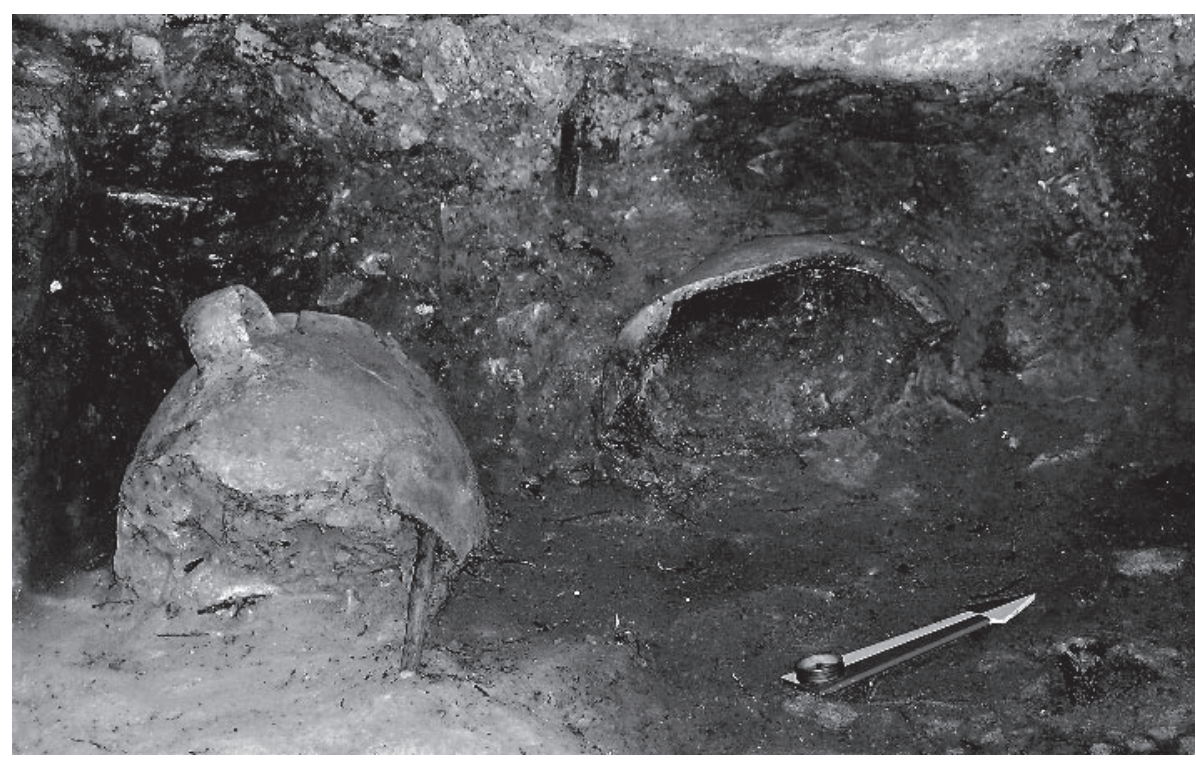

Fig. 18 - Les Baguarettes, vue du vase en place (photo : C. Gilabert).

Fig. 18 - Les Baguarettes, view of the vase in situ. 
Ces derniers sont très peu nombreux et appartiennent à des secteurs anatomiques variés (membre supérieur droit, ceintures du membre supérieur et inférieur, squelette axial...), à savoir :

- un grand fragment de diaphyse radiale gauche;

- un fragment latéral de diaphyse claviculaire gauche;

- divers fragments du sacrum (ailerons);

- divers fragments vertébraux (corps et lames);

- quelques fragments de coxaux, dont un fragment de la jonction ischio-iliaque en cours de synostose;

- et une incisive présentant une attrition importante.

Le déficit par rapport à un squelette complet est considérable et il n'est pas totalement impensable, non plus, que ces os appartiennent à plusieurs individus. L'apparente discordance entre l'usure dentaire et le stade d'épiphysation du fragment de coxal pourrait plaider en faveur de cela, mais la coexistence des deux caractères n'est pas inconcevable non plus. On retiendra donc que les os renvoient à au moins un adolescent.

Le vase (fig. 19) appartient à une catégorie moyenne ( $27 \mathrm{~cm}$ de haut, $22,5 \mathrm{~cm}$ de diamètre maximal environ). Sa partie supérieure est convergente (l'indice d'ouverture est de 0,761). Elle a une délinéation légèrement sinueuse et se termine par une lèvre aplatie. Le diamètre à l'ouverture est de $17,5 \mathrm{~cm}$ au maximum, le vase présente toutefois une ouverture irrégulière dont la forme est plus proche de l'ellipse que du cercle. Le fond, plat, mesure quant à lui $11 \mathrm{~cm}$ de diamètre. Ce récipient est également doté de deux anses en ruban, aux profils cintrés, prenant place symétriquement au niveau du point de segmentation externe.

Ce pot a été modelé dans une argile contenant naturellement de fins nodules d'oxyde de fer, à laquelle on a rajouté de la calcite pilée de taille moyenne. Le fond a vraisemblablement été modelé à partir d'une motte d'argile ou d'un colombin entouré sur lui-même. Pour la panse, les tranches de certains tessons montrent des configurations en $\mathrm{S}$, caractéristiques du montage au

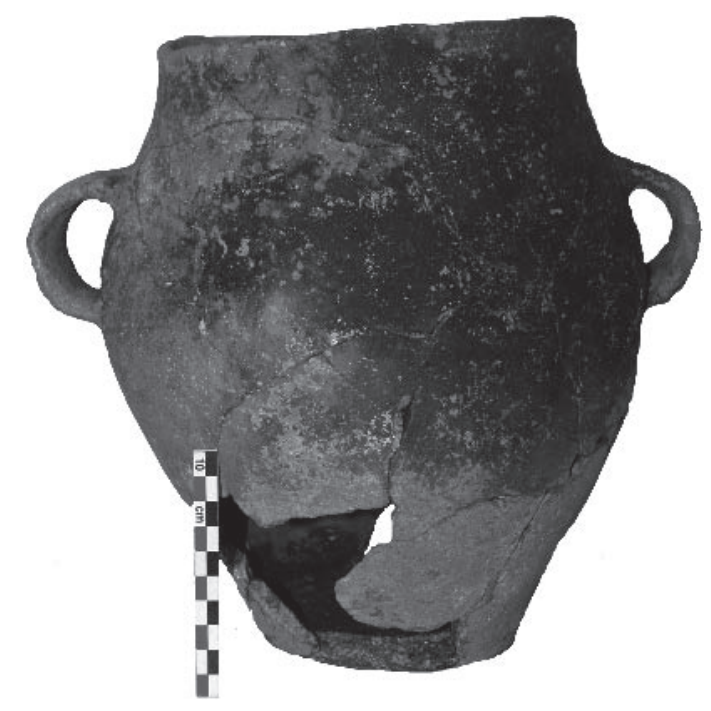

Fig. 19 - Les Baguarettes, le vase (photo : T. Lachenal) Fig. 19 - Les Baguarettes, the vase. colombin alterné, lissé successivement à l'intérieur puis à l'extérieur. Ce récipient a bénéficié d'une cuisson en atmosphère oxydante.

Les comparaisons les plus proches concernent le Languedoc et les gorges de la Cèze : la grotte du Baptème (Gard) et la Baume Resconduda (Ardèche) (Guy et Roudil, 1994; Lachenal, 2004; Roudil et Tscherter, 1995). En Provence, la rareté des témoins attribués à cette phase ayant fait l'objet de publication explique probablement le manque de comparaisons possibles. Tout juste peut-on évoquer des similitudes morphologiques avec des jarres de dimensions supérieures provenant de l'aven de la Mort de Lambert à Valbonne (Alpes-Maritimes) (étude T. Lachenal). Plus au nord, des parallèles sont possibles avec du mobilier provenant de la Baume des Anges à Donzère (Drôme), également daté du Bronze ancien (Vital, 1990, fig. 10, $\mathrm{n}^{\text {os }} 1,2,8$ et 2004 , p. 20 et 21, fig. 4). Des récipients de formes équivalentes se retrouvent aussi en Auvergne à la même période, comme à La carrière Malvy (DalletMachal, Puy-de-Dôme) ou au Tourteix (Orcet, Puy-deDôme) (Loison, 2003, fig. 25 et 81). Ce bref tour d'horizon permet de proposer une datation de la seconde moitié du Bronze ancien pour ce type de forme, car elle semble absente des productions antérieures.

Cependant, la datation ${ }^{14} \mathrm{C}$ réalisée depuis couvre plutôt, de manière un peu inattendue, la première phase du Bronze ancien, soit : Ly-4833 : 3555 × 30 BP : 2020 à 1980 av. n. è.

L'interprétation générale est délicate dans un contexte aussi altéré. En premier lieu, il est probable qu'il existe une relation entre les vestiges retrouvés, pour partie dans le vase, mais elle n'est pas certaine. Si l'on admet cette relation, elle peut être d'au moins deux natures. Il peut, soit s'agir d'une sépulture secondaire partielle en vase, soit d'une sépulture primaire extrêmement remaniée qui possédait ce vase en qualité de mobilier. Néanmoins, il n'est pas impensable que ces vestiges soient déconnectés en termes d'épisode... Si nous avons choisi de retenir cet ensemble, c'est qu'il évoque des manifestations funéraires bien documentées dans des nécropoles en cavités des Alpes italiennes à la même époque (cf. infra).

\section{LES COMPARAISONS}

\section{Les sépultures individuelles}

Comme mentionné dans l'introduction, les sépultures individuelles en fosses sont extrêmement rares en Languedoc. D'ailleurs celles en coffre le sont également, ces architectures étant, de plus, difficilement datables : il peut toujours s'agir de réemplois. Ce qui ne veut pas dire que de petits coffres n'ont pas été réalisés pendant le Bronze ancien, comme cela semble être établi pour les Causses (Gascó, 2004; ThauvinBoulestin, 1998, p. 40 à 46 et p. 76 à 78), les contreforts pyrénéens (Gardes, 1996, p. 546 et 547) et le bassin rhodanien (Tchérémissinoff, 2006).

Concernant les fosses, pour le Toulousain, on peut juste évoquer une tombe en silo sur le site de Villeneuve- 
Tolosane (information orale J. Vaquer). Cette configuration est également celle d'une sépulture se rapportant plutôt au Bronze ancien I, découverte au Roc d'en Gabit sur la commune de Carcassonne dans l'Aude. Les données disponibles évoquent une tombe d'enfant trouvée à la base d'un silo dans l'aire interne de l'enceinte (Vaquer et al., 2004, p. 482).

À une plus large échelle, les sépultures en silos demeurent également très rares. Seule la moyenne vallée du Rhône, avec le site à vocation domestique du Bronze ancien I de Chabrillan «Saint Martin 3» (Drôme) a livré trois sépultures de ce type (Blaizot et Rimbault, 2005). Quelques dépôts en fosses domestiques sont également recensés en Auvergne (Loison, 2003) où ils ont une existence ponctuelle et côtoient aussi sporadiquement des ensembles de tombes architecturées.

Pour les sépultures présentées, la qualification des fosses n'est pas aisée, voire impossible (Cépie, Canségala). Il faut noter toutefois que la sépulture du Bénazet a probablement fait l'objet d'une protection rigide, de type coffre ou coffrage en matériau périssable.

Là encore, les comparaisons sont rares et éloignées. Elles concernent le site des Julliéras (Mondragon, Vaucluse) en basse vallée du Rhône (Lemercier et al., 1998, p. 359 à 368 et 2002, p. 61 à 66). Pour ce petit ensemble (ou sous-ensemble d'une nécropole plus étendue), l'étude des neuf sépultures trahit ce type de dispositif.

D'une manière générale, pour la vallée du Rhône (Tchérémissinoff, 2006) et la Provence (Lemercier et al., 2004), les données contredisent pour l'instant l'image d'un retour tranché vers la sépulture individuelle au Bronze ancien, voire au Bronze moyen. Ce constat paraît être le même pour le Midi méditerranéen (Lemercier et Tchérémissinoff, à paraître). Elle pourrait néanmoins peut-être émerger progressivement à la faveur, notamment, de grandes séries de datations sur les sépultures simples des grandes zones à occupations successives des plaines alluviales.

Pour la période considérée, les deux ensembles de référence ont été fouillés dans le Puy-de-Dôme ${ }^{2}$. Eux seuls peuvent soutenir le qualificatif de nécropole, bien qu'ils présentent des configurations différentes.

En effet, le site de la carrière Malvy à Dallet-Machal (Loison, 2003) a livré une trentaine de sépultures se scindant en sous-ensembles, contigus voire mêlés à un habitat contemporain se rattachant plutôt à la seconde phase du Bronze ancien. Alors que la nécropole de Chantemerle à Gerzat (Vermeulen et al., 2002), peutêtre fondée un peu plus anciennement, est une zone sépulcrale exclusive qui a livré soixante-douze sépultures. Mise à part la présence d'enclos funéraires sur ce site, les deux ensembles offrent des choix architecturaux comparables. Le dispositif dominant est celui d'un coffre ou coffrage en bois, déposé dans une large fosse, puis bloqué par un dispositif plus ou moins important de pierres ou de dalles qui sert d'assise à un tertre également en pierres. On observe quelques coffres simples (non augmentés d'éléments lithiques), mais un autre choix nous intéresse en particulier, il se retrouve sur les deux sites : il s'agit de sépultures de périnataux et de nourrissons en jarre.

\section{Les sépultures \\ en jarres}

Malheureusement, en l'absence de publications monographiques, on ne connaît pas exactement leur nombre. En incluant d'autres sites auvergnats (Loison, 2003), il pourrait s'élever à une dizaine, voire plus. Pareillement, on ne dispose pas d'informations précises sur les modalités de sélection et de dépôt. L'un d'entre eux, au moins, était ceint d'un dispositif lithique (Loison, 2003, p. 22).

Les ensembles de références à ce sujet concernent des zones géographiques encore plus éloignées : la bordure méditerranéenne sud-orientale de l'Espagne et la vallée alpine de l'Adige en Italie.

Le Sud-Est de l'Espagne (provinces d'Almeria et de Murcie) demeure sans aucun doute la zone la plus documentée en ce qui concerne la pratique des sépultures en jarre.

C'est la fouille du site d'El Argar par les frères Siret, à la fin du XIX ${ }^{\mathrm{e}}$ siècle (Siret et Siret, 1887), qui a révélé de façon spectaculaire ce mode funéraire. Il s'agit d'un habitat installé sur un promontoire surmontant la plaine, entouré de murailles, dont l'occupation s'étire sur six siècles environ (2250 à 1650 av. n. è.). Il recelait plus d'un millier de sépultures : en petits hypogées, en diaclases aménagées, en caveaux architecturés, en coffres et en jarres (pithoi), à l'extérieur ou à l'intérieur des unités d'habitations. L'ensemble a livré un mobilier métallique extrêmement riche (parures, armes, outils, en alliage cuivreux et en argent principalement), mais aussi un abondant mobilier céramique, des objets de parures et du quotidien, qui semblent exclusivement destiné aux adultes $^{3}$. Les tombes en jarres, qui se développent durant la seconde phase de la culture d'El Argar (1900 à 1600 av. n. è., Bronze ancien II) concernent aussi des adultes, et les enfants (à partir d'un certain âge ?) peuvent aussi bénéficier d'autres contenants. Des dépôts pluriels en jarres sont également recensés. Ces contenants peuvent être disposés dans de simples fosses (architecturées ou non) ou de petits coffres lithiques. Les frères Siret sont également à l'origine de la découverte d'autres sites présentant les mêmes caractères défensifs, architecturaux et funéraires (Cauwe, 2003).

Une fouille plus récente à Los Cipreses (Lorca, Murcie), démontre que même en plus petit nombre, ces types funéraires se mêlent pareillement (Martínez Rodríguez et al., 1993). Il s'agit d'une implantation domestique, mais qui ne semble pas incluse dans un système défensif. La première intervention sur le site a révélé quatre maisons en pierres sèches et douze sépultures localisées entre ou à l'intérieur des bâtiments. Elles se composent de quatre coffres (cinq cistes à antennes formant des espaces externes pour le dépôt de mobiliers et un coffre multidallé ou mixte) et six tombes en jarres. Sur ce site, ce dernier mode concerne surtout des nourrissons, mais on recense aussi un adulte. Ce dernier ne bénéficie pas de mobilier ostensible, comme pour les défunts en coffre, auxquels sont associés poignards, bracelets, 
céramiques ${ }^{4} \ldots$ Un enfant d'environ deux ans a bénéficié lui d'un coffre multidallé, mais aucun mobilier ne lui est également associée. Les jarressépultures font toutes l'objet de calages plus ou moins élaborés et de dispositifs de fermeture et signalisation en pierres. Enfin, une jarre dont les os n'ont pu être étudiés recelait un second vase (mobilier? vase gigogne?). Une autre de ses particularités est de reposer au fond d'une fosse de plus d'un mètre de diamètre, qui pourrait être d'origine domestique. Elle dispose aussi d'un important dispositif de fermeture lithique.

Une petite synthèse sur les rites funéraires de la culture d'El Argar dans la région de Lorca (Martínez Rodríguez et al., 1996), qui porte sur une vingtaine de tombes, reprend les types déjà brièvement décrits, mais révèle aussi quelques particularismes. En premier lieu, la présence d'espaces sépulcraux réservés au sein de grandes fosses (caves? hypogées ?), puis empierrées, qui concernent des adultes avec des mobiliers standards, équivalents à celui des coffres. En second lieu, deux tombes à deux jarres accolées par leurs cols (doble urna), ici destinées à des adultes pourvus de mobilier.

Dans la région de Trento (Haut Adige), les choix d'implantation sont systématiquement les mêmes. Il s'agit de petites cavités, de boyaux ou de simples abris sous surplombs rocheux, auxquels on accède par d'étroites vallées convergeant vers l'Adige. Ces lieux recèlent de petits ensembles sépulcraux, sans doute à caractère plus familial que collectif, qui concernent des adultes et des enfants de tous âges. Les nourrissons sont principalement concernés par les dépôts en jarre, mais pas exclusivement. Des bordures de pierres semblent parfois aménagées, mais les chaos naturels de blocs sont aussi exploités pour délimiter des espaces sépulcraux, en jarres ou nus (espaces ouverts). La chronologie concerne là aussi un plein Bronze ancien, qui n'est peut-être pas antérieur à 2000 av. n. è.

L'ensemble le plus connu est celui de Romagnano Loc à Nogarole di Mezzolombardo (Perini, 1971 et 1975; Broglio et Perini, 1996; Nicolis, 1996 et 2004). Il a livré majoritairement des enfants, des jeunes enfants et des enfants morts en période périnatale. L'espace sépulcral occupait une surface d'environ $6 \mathrm{~m}^{2}$ contre une paroi rocheuse, sous son surplomb. Les dépôts sont surtout individuels. Ceux des adultes et des enfants les plus âgés ont été réalisés à même le sol ou bien dans de légères excavations. Ils se trouvaient limités et recouverts par de grandes pierres. Les restes étaient assez perturbés, comme lorsqu'ils sont restés longtemps accessibles. Les très jeunes enfants (périnataux et nourrissons) étaient contenus dans de grandes jarres de stockage, bordées de blocs et recouvertes de petites accumulations de pierres. Dans au moins un cas, une jarre contenait le crâne d'un enfant plus grand (env. 4-5 ans) et des dépôts secondaires très partiels d'adultes en vase sont connus dans d'autres ensembles, par exemple une côte sur le site de Riparo 3 et un crâne sur le site de Riparo del Santuaro (Nicolis, 2004).

\section{QUELLE ORIGINE POSSIBLE POUR LA SÉPULTURE EN JARRE DE CANSÉGALA?}

\section{Une genèse méditerranéenne dans les cultures du Bronze ancien?}

Il est compliqué de définir l'antériorité de la manifestation d'un domaine culturel cité par rapport à l'autre, car la fourchette chronologique semble être toujours la même, elle se rapporte à un plein Bronze ancien $\left(1900\right.$ à 1650 av. n. è. environ $\left.{ }^{5}\right)$. Des aires culturelles centre-européennes semblent concernées par cette pratique (cité par Loison, 2003, p. 126), mais nous n'avons pas pu accéder aux sources bibliographiques originales. Quant à la Méditerranée orientale, souvent évoquée par les auteurs comme origine possible et à laquelle le terme de tombe en pithos du domaine argarique fait d'ailleurs clairement référence, les données relatives à des contacts concrets ne sont pas antérieures au XIII ${ }^{\mathrm{e}}$ siècle av. n. è. (Warmenbol, 2003).

L'autre problème réside dans le fait que les appropriations stylistiques se produisent à une large échelle dans une marge temporelle au sein de laquelle les successions ne sont que peu décelables par $1{ }^{14} \mathrm{C}$. Ainsi, si l'origine des décors en arceaux de la jarre de Canségala réside probablement dans le substrat campaniforme du domaine atlantique, au Bronze ancien évolué ils se retrouvent aussi bien en basse vallée du Rhône et en Languedoc. De même, le vase du Travers des Baguarettes relève d'une large répartition, puisqu'il évoque à la fois le Languedoc, l'Auvergne, la moyenne vallée du Rhône et le domaine italique, ce dernier présentant peut-être une légère antériorité. Seules les productions d'El Argar semblent un peu à l'écart (Cauwe, 2003), inscrites dans d'autres réseaux, alors que ceux-ci demeurent entre les productions de la Catalogne, de l'Aragon et le Midi de la France (Agustí et al., 1997; Guilaine, 1972 et 1996).

Pour ces raisons, s'il fallait proposer une origine, le domaine poladien aurait notre préférence et, dans ce cas, il serait effectivement peut-être intéressant de regarder vers l'Europe centrale (Vital, 2004, p. 32), plutôt que vers le bassin oriental de la Méditerranée.

\section{Une genèse dans le substrat chalcolithique régional?}

Cependant, les dépôts d'enfants en jarres ne sont pas totalement inconnus du substrat chalcolithique régional.

En effet, bien que cette sépulture n'ait jamais été publiée en détail, le contexte de dépôt d'un nourrisson sur l'habitat fontbuxien de Cambous (Hérault) ne fait pas de doute. Il était contenu dans un vase (une marmite hémisphérique), installé dans un locus entre deux maisons, comme dans le cas des sépultures argariques (Bordreuil, 1985, p. 31 et 32; Canet et al., 1978, p. 173 et 174). 
Un autre témoin récemment fouillé vient enrichir la proposition d'une origine locale.

Il s'agit d'une sépulture concernant deux sujets, qui a été découverte sur le site fontbuxien de la Capoulière à Mauguio dans l'Hérault (Gutherz et Jallot, 2004). Ils étaient scellés dans le comblement inférieur d'une grande fosse domestique. Il s'agit d'un adulte et d'un enfant d'environ 6 ans. Ce dernier était contenu dans une grande jarre de stockage, couchée à l'horizontale. Il était fléchi sur le côté gauche, son crâne outrepassant nettement le contenant. Les deux défunts ont été disposés à proximité de la paroi, respectivement au nordest et au sud-est. Il n'y a donc pas de contact entre eux, mais leur dépôt a eu lieu durant une même phase de remblaiement (Cros, 2004, p. 69 à 74).

\section{Une genèse multipolaire?}

Si les dépôts d'enfants en jarres sont connus dans le substrat chalcolithique, ils ne rencontrent a priori pas le succès remporté dans les zones citées pour le Bronze ancien évolué. Il faut aussi relever qu'un important hiatus subsiste pour ce type de tombes durant les cinq à six siècles couvrant le Campaniforme et le Bronze ancien I, même à une large échelle. Pour le Sud de la France, ce sont d'ailleurs aussi les habitats stricto sensu de cette période qui font défaut et, nous l'avons vu, ces pratiques sont intimement liées aux espaces domestiques.
Il n'est naturellement pas possible de conclure en l'état. Mais l'utilisation d'un vase, comme «matrice» d'inhumation pour les jeunes enfants a pu émerger sporadiquement avant d'être réellement ritualisée à partir de nouvelles influences au cours du développement du plein Bronze ancien.

Remerciements : Merci infiniment à Jean-Paul Cazes et Christophe Gilabert, pour avoir bien voulu partager leurs données et nous faire confiance quant à leur diffusion.

Un grand merci également à Jean-Paul Cros, Luc Jallot et Xavier Gutherz pour les documents concernant la sépulture de la Capoulière et pour nous avoir permis d'évoquer ces données, alors inédites.

\section{NOTES}

(1) Toutes les dates présentées dans cet article sont calibrées à 2 sigmas (cal. 95,4\% de probabilités) avec Oxcal V3.10.; cub r : 5 sd : 12 prob usp[chron].

(2) Depuis le dépôt de l'article (novembre 2007), un troisième ensemble a été mis au jour, à Lemdes dans le Puy-de Dôme (resp. P. Hénon, Inrap).

(3) La publication originale est disponible sur Internet aux adresses suivantes : http://www.arqueomurcia.com/index.php?a=pu_libro_siret et http://www.arqueomurcia.com/index.php?a=pu_album_siret

(4) Cela est loin d'être une systématique sur le site éponyme, où, par exemple, la fameuse tombe au diadème (62), concerne un adulte déposé en jarre.

(5) Il faut dire que les calibrations font presque toujours défaut pour les dates des sites espagnols et italiens.

\section{RÉFÉRENCES BIBLIOGRAPHIQUES}

AGUSTÍ B., ALONSO N., BORRÀS H., BRUGUERA R., FERRER C. GOMIS M., GONZALO C., JUNYENT E., LAFUENTE A. LÓPEZ J.B., NOGUERA J., LLUSSÀ A., MAZO C., MIRADA J., MIRÓ J.M., MORÁN M., REY J., ROVIRA C., ROVIRA N., SAULA I O., TARTERA E. (1997) - Noves dades per a la caracterització dels assentaments a l'aire lliure durant la primera meitat del II mil.lenni cal. BC: primers resultatsde les excavacions en el jaciment de Minferri (Juneda, les Guarrigues), Revista d'Arqueologia de Ponent, $\mathrm{n}^{\circ} 7$, p. 161-211.

BAILLS H. (1995) - La grotte de la Chance et l'Âge du bronze moyen en Conflent, Cultures i medi de la Prehistória a l'Edat Mitjana. Homenatge al Professor Jean Guilaine, $X^{e}$ Colloqui Internacional d'Arqueologia de Puigcerdá, Puigcerdá et Osseja, 10-12 nov. 1994, p. 367 375.

BILLARD C., BLANCHET J.-C., TALON M. (1996) - Origine et composantes de l'Âge du bronze ancien dans le Nord-Ouest de la France, in $\mathrm{C}$. Mordant et O. Gaiffe dir., Cultures et sociétés du Bronze ancien en Europe, Actes du $117^{e}$ congrès national des Sociétés savantes, Clermont-Ferrand, 27-29 oct. 1992, éd. du CTHS, Paris, p. $579-602$.

BLAIZOT F., RIMBAULT S. (2005) - Des inhumations en contexte domestique au Bronze ancien en Rhône et Auvergne, in $\mathrm{C}$. Mordant et $\mathrm{G}$. Depierre dir., Les pratiques funéraires à l'Âge du bronze en France, Actes de la table ronde de Sens-en-Bourgogne (Yonne), éd. du CTHS et de la Société archéologique de Sens, Paris et Sens, p. 345363.

BORDREUIL M. (1985) - Rites funéraires de l'Âge du cuivre en Languedoc oriental, Actes du $110^{\mathrm{e}}$ Congrès national des Sociétés savantes, Archéologie, Montpellier, p. 23-36.

BROGLIO A., PERINI R. (1996) - Romagnano III, Preistoria e Protostoria, Guide Archeologiche, $n^{\circ} 4$, Congresso Internazionale delle Scienze Prehistoriche e Protostoriche, Forli, Italie, p. 102-118.
BRUZEK J. (1991) - Fiabilité des procédés de détermination du sexe à partir de l'os coxal. Implications à l'étude du dimorphisme sexuel de l'homme fossile, thèse de doctorat du Muséum national d'histoire naturelle, Institut de paléontologie humaine.

BRUZEK J. (2002) - A method for visual determination of sex, using the human hip bone, American Journal of Physical Anthropology, t. 117 , p. $157-168$.

CANET H., POULAIN R., ROUDIL J.-L. (1978) - Le village chalcolithique de Cambous à Viol-en-Laval (Hérault), Gallia Préhistoire, t. 21, p. 143-188.

CAUWE N. dir. (2003) - Un Âge d'argent. Premiers agriculteurs et premiers métallurgistes dans le Sud-Est de l'Espagne, La collection "Siret» des musées d'Art et d'Histoire, musée du Malgré-Tout, Treignes, $63 \mathrm{p}$.

CAZES J.-P. dir. (2005) - La nécropole mérovingienne de Bénazet, commune de Molandier (Aude), rapport de fouille programmée, SRA Languedoc-Roussillon, Montpellier.

CLAUSTRE F. (1996) - Le Bronze ancien en Roussillon, in C. Mordant et O. Gaiffe dir., Cultures et sociétés du Bronze ancien en Europe, Actes du $117^{e}$ congrès national des Sociétés savantes, ClermontFerrand, 27-29 oct. 1992, éd. du CTHS, Paris, p. 387-399.

CLEUVENOT E., HOUËT F. (1993) - Proposition de nouvelles équations d'estimation de stature applicables pour un sexe indéterminé, et basées sur les échantillons de Trotter et Gleser, Bulletins et mémoires de la Société d'anthropologie de Paris, n. s., n ${ }^{\circ}$ 5, p. 245-255.

CROS J.-P. (2004) - Première étude anthropologique des inhumations de la fosse 4005, in X. Gutherz et L. Jallot dir., Recherche archéologique programmée : le Néolithique de Mauguio, La Capoulière IV (Hérault), rapport de fouilles programmées, SRA LanguedocRoussillon, Montpellier, p. 69-74. 
GARDES P. (1996) - Les piémonts pyrénéens occidentaux à la charnière du Néolithique, in C. Mordant et O. Gaiffe dir., Cultures et sociétés du Bronze ancien en Europe, Actes du $117^{e}$ congrès national des Sociétés savantes, Clermont-Ferrand, 27-29 oct. 1992, éd. du CTHS, Paris, p. 539-561.

GASCÓ J. (2004) - Les composantes de l'Âge du bronze, de la fin du Chalcolithique à l'Âge du Bronze ancien, Cypsela, t. 15, p. 39-72.

GASCÓ J., CAROZZA L., WAINWRIGHT J. (1996) - Un petit habitat agricole de l'Âge du Bronze ancien en Languedoc occidental : Laval de la Bretonne (Monze, Aude), hypothèses et conséquences d'un enfouissement sur la «courte durée» de l'occupation humaine, in C. Mordant et O. Gaiffe dir., Cultures et sociétés du Bronze ancien en Europe, Actes du $117^{e}$ congrès national des Sociétés savantes, Clermont-Ferrand, 27-29 oct. 1992, éd. du CTHS, Paris, p. 373385 .

GILABERT C., THIBAULT T., THOMANN A. (2006) - Le Travers des Baguarettes (Ménerbes, Vaucluse), rapport de fouille de sauvetage, $20 \mathrm{p}$.

GIRAUD J.-P., VAQUER J. (1994) - Un possible rebut de production céramique de l'Âge du bronze moyen à Villeneuve-Tolosane (HauteGaronne), Terre cuite et société. La céramique, document technique, économique, culturel, XIV ${ }^{\text {s }}$ Rencontres internationales d'archéologie et d'histoire d'Antibes, Juan-les-Pins, éd. APDCA, Antibes, p. 217 224.

GOMEZ de SOTO J. (1995) - Le Bronze moyen en Occident, L’Âge du bronze en France, t. 5, éd. Picard, Paris, 375 p.

GOMEZ de SOTO J., JOUSSAUME R., PAUTREAU J.-P. (1996) - Le Bronze ancien au sud de la Loire, in C. Mordant et O. Gaiffe dir., Cultures et sociétés du Bronze ancien en Europe, Actes du 117 congrès national des Sociétés savantes, Clermont-Ferrand, 27-29 oct. 1992, éd. du CTHS, Paris, p. 499-501.

GUILAINE J. (1972) - L'Âge du bronze en Languedoc occidental, Roussillon, Ariège, Mémoire de la Société préhistorique française, t. 9, éd. Klincksieck, Paris, 460 p.

GUILAINE J. (1996) - Le Bronze ancien en Méditerranée occidentale, in C. Mordant et O. Gaiffe dir., Cultures et sociétés du Bronze ancien en Europe, Actes du $117^{e}$ congrès national des Sociétés savantes, Clermont-Ferrand, 27-29 oct. 1992, éd. du CTHS, Paris, p. 37-68.

GUTHERZ X., JALLOT L. dir. (2004) - Recherche archéologique programmée : le Néolithique de Mauguio, La Capoulière IV (Hérault), rapport de fouilles programmées, SRA Languedoc-Roussillon, Montpellier, $90 \mathrm{p}$.

GUY B., ROUDIL J.-L. (1994) - Une grotte sépulcrale inédite de la Cèze : la Baume Resconduda, Ardèche Archéologie, t. 11, p. 7-11.

HAUSER G., DE STEFANO G.F. (1989) - Epigenetic variants of the human skull, Schweizerbart, Stuttgart.

LACHENAL T. (2004) - Le Bronze ancien évolué des Iragnons-Perrier Codognan (Gard), Étude morpho-typologique de la céramique des fosses, mémoire de maîtrise de l'université de Provence, Aix-enProvence, $194 \mathrm{p}$.

LEMERCIER O., DÜH P., LOIRAT D., MELLONY P., PELISSIER M., SERIS D., TCHÉRÉMISSINOFF Y., BERGER J.-F. (1998) - Les Juilléras (Mondragon, Vaucluse). Site d'habitat et funéraire du Néolithique récent, Néolithique final, Campaniforme - Bronze ancien e Bronze final $2 \mathrm{~b}$ : premiers résultats, in A. D'Anna et D. Binder dir. Production et identité culturelle, Actes des Rencontres méridionales de préhistoire récente, $2^{e}$ session, Arles, novembre 1996, éd. APDCA, Antibes, p. 359-368.

LEMERCIER O., PELISSIER M., TCHÉRÉMISSINOFF Y. (2004) Campaniforme et sépultures, au-delà du standard. La place du Campaniforme dans l'évolution des sépultures du sud-est de la France au $3^{\mathrm{e}}$ millénaire avant notre ère, in M. Besse et J. Desideri dir., Graves and Funerary Rituals during the Late Neolithic and the Early Bronze Age in Europe (2700-2000 BC), Proceedings of the International Conference held at Cantonal Archaeological Museum, Sion (Switzerland), du 4 au 7 octobre 2001, BAR International Series 1284, Oxford, p. $49-59$.
LEMERCIER O., TCHÉRÉMISSINOFF Y, avec la coll. de PELISSIER M., FURESTIER R. (2002) - Les Juilléras (Mondragon), in J. Buisson-Catil et J. Vital dir., L'Âge du bronze en Vaucluse, Notice d'archéologie vauclusienne, $\mathrm{n}^{\circ} 5$, Travaux du Centre d'archéologie préhistorique de Valence, Avignon, p. 61-66.

LEMERCIER O., TCHÉRÉMISSINOFFY. (à paraître) - Du Néolithique final au Bronze ancien : les sépultures individuelles campaniformes dans le sud de la France, in L. Salanova et Y. Tchérémissinoff dir., Les sépultures individuelles campaniformes en France, Gallia Préhistoire.

LOISON G. (2003) - L'Âge du Bronze ancien en Auvergne, Archives d'écologie préhistorique, $\mathrm{n}^{\circ} 14$, École des hautes études en sciences sociales, Toulouse, $156 \mathrm{p}$.

LOVEJOY C. O. (1985) - Dental wear in the Libben population: Its functional pattern and role in the determination of adult skeletal age at death, American Journal of Physical Anthropology, t. 68, p. 47-56.

LOVEJOY C. O., MEINDL R.S., PRYSBECK T.R., MENSFORTH R.P. (1985) - Chronological metamorphosis of the auricular surface of the ilium: a new method for the determination of adult skeletal age at death, American Journal of Physical Anthropology, t. 68, p. 15-28.

MARTINEZ RODRIGUEZ A., PONCE GARCIA J., MANUELA AYALA JUAN M. (1993) - Excavaciones de urgencia del poblado argárico de los Cipreses, Lorca, años 1992-1993, Memorias de Arqueología, p. 156-182.

MARTINEZ RODRIGUEZ A., PONCE GARCIA J., MANUELA AYALA JUAN M. (1996) - Las práticas funerarias de la cultura argárica en Lorca-Murcía, éd. Caja de ahorros de Murcia et Excmo, Ayuntamiento de Lorca, $36 \mathrm{p}$.

NICOLIS F. (1996) - Strutturi e riti funebri : L'Italia settentrionale, L'antica età del bronzo, Atti del Congresso di Viareggo, 9-12 juin 1995, Firenze, p. 337-344.

NICOLIS F. (2004) - Le evidenze funerarie dell' antica età del bronzo in Italia settentrionale, in M. Besse et J. Desideri dir., Graves and Funerary Rituals During the Late Neolithic and the Early Bronze Age in Europe (2700-2000 BC), Proceedings of the International Conference held at Cantonal Archaeological Museum, Sion (Switzerland), 4-7 octobre 2001, BAR International Series 1284, Oxford, p. 111145 .

OLIVIER G. (1960) - Précis de pratique anthropologique, éd. Vigot frères, Paris.

OWINGS-WEBB P.A., SUCHEY J.M. (1985) - Epiphyseal union of anterior iliac crest and medial clavicle in a modern multiracial sample of American males and females, American Journal of Physical Anthropology, t. 68, p. 457-466.

PERINI R. (1971) - I depositi preistorici di Romagno Loc (Trento), Preistoria Alpina, t. 7, p. 7-106.

PERINI R. (1975) - La necropoli di Romagno - Loc III e IV, Le tombe all' inizio dell' età del Bronzo nella regione Sudalpina Centroorientale, Preistoria Alpina, t. 11, p. 295-315.

PONS F., LAGARRIGUE A., COLONGE D., JARRY M., MARTIN H. (2003) - Un habitat de plein air du Bronze ancien dans le Midi toulousain à Blagnac (Haute-Garonne) : le site de Cassagna 3, Documents d'archéologie méridionale, $\mathrm{n}^{\circ}$ 26, Lattes, p. 7-41.

ROUDIL J.-L., TSCHERTER E. (1995) - La grotte sépulcrale du Baptème (gorges de la Cèze), Ardèche Archéologie, t. 12, p. 29-38.

ROUQUEROL N. (2004) - Du Néolithique à l'Âge du bronze dans les Pyrénées centrales françaises, Archives d'écologie préhistorique, $\mathrm{n}^{\circ} 16$, École des hautes études en sciences sociales, Toulouse, $187 \mathrm{p}$.

SIRET H., SIRET L. (1887) - Les premiers Âges du métal dans le SudEst de l'Espagne, éd. Andwerpen, 3 vol., 437 p.

SJOVOLD T. (1984) - A report on the heritability of some cranial measurements and non-metric traits, in G.N. Van Vark et W.W. Howells dir., Multivariate statistical methods in physical anthropology, D. Reidel publishing company, p. 223-246. 
STLOUKAL M., HANAKOVA H. (1978) - The length of long bones in ancient Slavonic populations, with particular consideration to the questions of growth, Homo, t. 29, p. 53-69.

TCHÉRÉMISSINOFF Y. (2003) - Deux nouvelles sépultures dans la région de Limoux (Aude), in P. Chambon et J. Leclerc dir., Pratiques funéraires du Néolithique ancien et moyen en France et dans les régions limitrophes entre 5000 et 3500 environ av. J.-C., Actes de la table ronde de Saint-Germain-en Laye, 15 et 16 et juin 2001, Mémoire de la Société préhistorique française, t. 33, p. 185-189.

TCHÉRÉMISSINOFF Y. (2006) - Les sépultures simples et plurielles du Campaniforme et du Bronze ancien dans le Bassin rhodanien et ses zones d'influences, BAR International Series 1531, Oxford, $155 \mathrm{p}$.

THAUVIN-BOULESTIN E. (1998) - Le Bronze ancien et moyen des Grands Causses et des Causses du Quercy, Documents préhistoriques, $\mathrm{n}^{\circ} 11$, éd. du CTHS, Paris, 335 p.

UBELAKER D.H. (1989) - Human skeletal remains. Excavations, analysis, interpretations, Manuels on Archaeology, édition révisée, Taraxacum, Washington.

VAQUER J., GANDELIN M., MARSAC R. (2004) - Le site du Roc d'en Gabit, Carcassonne (Aude), in H. Dartevelle dir., Auvergne et Midi, Actualité de la recherche, Actes de la $5^{e}$ session des Rencontres méridionales de préhistoire récente, 8-9 novembre 2002, éd. Préhistoire du Sud-Ouest, Clermont-Ferrand, p. 475-484.

VERMEULEN C., CABEZUELO U., LISFANC R. (2002) - Une nécropole de l'Âge du bronze, L'Archéologue, n 59, p. 48-49.

VITAL J. (1990) - Protohistoire du défilé de Donzère : l'Âge du bronze dans la Baume des Anges (Drôme), Documents d'archéologie française, $\mathrm{n}^{\circ} 28$, éd. Maison des sciences de l'homme, Paris, $152 \mathrm{p}$.

VITAL J. (2004) - Du Néolithique final au Bronze final dans le Sud-Est de la France : 2200-1300 av. J.-C., Cypsela, t. XV, p. 11-38.

WARMENBOL E. (2003) - La naissance de la métallurgie en péninsule Ibérique, in N. Cauwe dir., Un Âge d'argent. Premiers agriculteurs et premiers métallurgistes dans le Sud-Est de l'Espagne. La collection "Siret» des musées d'Art et d'Histoire, musée du Malgré-Tout, Treignes, p. 35-56.

Yaramila TCHÉRÉMISSINOFF Inrap - Méditerranée et CRPPM UMR 5608

54, avenue du Pont-Juvénal, 34000 Montpellier yaramila.tcheremissinoff@inrap.fr
Jean-Paul CAZES

CCS Patrimoine

47, rue Château-d'Eau, 11400 Castelnaudary jeanpaul.cazes@wanadoo.fr

Christophe GILABERT

Direction régionale des affaires culturelles de

Champagne-Ardenne

Service régional de l'Archéologie

3, faubourg Saint-Antoine 51037 Châlons-en-Champagne Cedex christophe.gilabert@culture.gouv.fr

Sylvie DUCHESNE Inrap - Grand Sud Ouest Base Inrap

ZI Les Champs Pinsons, 13, rue du Négoce 31650 Saint-Orens-de-Gameville sylvie.duchesne@inrap.fr

Thierry LACHENAL

Doctorant

Université d'Aix-en-Provence

Maison méditerranéenne des sciences de l'homme,

5, rue du Château-de-l'Horloge, BP 647, 13094 Aix-en-Provence Cedex 2

lachenal@mmsh.univ-aix.fr

Anne LAGARRIGUE Inrap - Grand Sud Ouest

Base Inrap

ZI Les Champs Pinsons, 13, rue du Négoce 31650 Saint-Orens-de-Gameville anne.lagarrigue@inrap.fr

Delphine MARET

Faculté dentaire

3, chemin des maraîchers

31062 Toulouse Cedex delphine_maret@yahoo.fr 